\title{
Molecular insights into biogenesis of glycosylphosphatidylinositol anchor proteins
}

\section{Yidan Xu}

State Key Laboratory of Molecular Biology, CAS Center for Excellence in Molecular Cell Science, Shanghai Institute of Biochemistry and Cell Biology, University of CAS, Chinese Academy of Sciences

\section{Guowen Jia}

The State Key Laboratory of Biotherapy and Cancer Center, Department of Geriatrics and National Clinical Research Center for Geriatrics, West China Hospital, Sichuan University

\section{Tingting Li}

University of Chinese Academy of Sciences https://orcid.org/0000-0003-3146-2761

\section{Zixuan Zhou}

Shanghai Stomatological Hospital, School of Stomatology, Shanghai Key Laboratory of Medical Epigenetics, International Co-laboratory of Medical Epigenetics and Metabolism, Fudan University

\section{Yitian Luo}

ShanghaiTech University https://orcid.org/0000-0001-7808-9322

\section{Yulin Chao}

Shanghai Stomatological Hospital, School of Stomatology, Shanghai Key Laboratory of Medical Epigenetics, International Co-laboratory of Medical Epigenetics and Metabolism, Fudan University Juan Bao

Shanghai Institute of Biochemistry and Cell Biology, University of Chinese Academy of Sciences

\section{Zhaoming Su}

Sichuan University https://orcid.org/0000-0002-9279-1721

\section{Qianhui Qu}

Stanford University

\section{Dianfan Li ( $\sim$ dianfan.li@sibcb.ac.cn )}

State Key Laboratory of Molecular Biology, CAS Center for Excellence in Molecular Cell Science, Shanghai Institute of Biochemistry and Cell Biology, University of CAS, Chinese Academy of Sciences https://orcid.org/0000-0003-4729-4678

\section{Article}

\section{Keywords:}

Posted Date: March 1st, 2022 
DOI: https://doi.org/10.21203/rs.3.rs-1339620/v1

License: (c) (1) This work is licensed under a Creative Commons Attribution 4.0 International License. Read Full License

Version of Record: A version of this preprint was published at Nature Communications on May 12th, 2022. See the published version at https://doi.org/10.1038/s41467-022-30250-6. 

glycosylphosphatidylinositol anchor proteins

Authors: Yidan $\mathrm{Xu}^{1, \dagger}$, Guowen $\mathrm{Jia}^{2, \dagger}$, Tingting $\mathrm{Li}^{1,}{ }^{\dagger}$, Zixuan $\mathrm{Zhou}^{3, \dagger}$, Yitian Luo ${ }^{1}$, Yulin $\mathrm{Chao}^{3}$, Juan $\mathrm{Bao}^{1}$, Zhaoming $\mathrm{Su}^{2, *}$, Qianhui $\mathrm{Qu}^{3, *}$, Dianfan $\mathrm{Li}^{1, *}$

\section{Affiliations:}

${ }^{1}$ State Key Laboratory of Molecular Biology, CAS Center for Excellence in Molecular

Cell Science, Shanghai Institute of Biochemistry and Cell Biology, University of CAS,

Chinese Academy of Sciences (CAS), 320 Yueyang Road, Shanghai 200030, China.

${ }^{2}$ State Key Laboratory of Biotherapy and Cancer Center, Department of Geriatrics and

Lead contact: dianfan.li@ sibcb.ac.cn 


\section{Abstract}

Eukaryotic cells are coated with an abundance of glycosylphosphatidylinositol anchor proteins (GPI-APs) that play crucial roles in fertilization, neurogenesis, and immunity. Covalent addition of structurally diverse GPI anchorages at the carboxyl termini of target proteins is catalyzed by an endoplasmic reticulum integral membrane GPI transamidase complex (GPI-T) conserved among all eukaryotes. Despite its important role in developmental and cancer biology, a detailed picture of this intricate multicomponent machinery remains elusive. Here, we report the cryo-electron microscopy (EM) structure of the human GPI-T complex at a global $2.53 \AA$ resolution, revealing an assembly mechanism whereby the catalytic subunit PIGK and its catalytic dyad are optimally positioned to accommodate its characteristic amphipathic substrates. Structural and functional characterizations suggest a previously unrecognized composite GPI-binding site formed by subunits PIGU/PIGK/PIGT and rationalize genetic diseases associated with GPI-T point mutations. Our work presents an important step towards the mechanistic understanding of GPI-AP biosynthesis. 


\section{Introduction}

The GPI anchoring represents a ubiquitous, metabolically expensive posttranslational modification of eukaryotic cell surface proteins ${ }^{1-5}$. Structurally elucidated in the 1980 s $^{6}$, GPI lipids are bioactive ${ }^{7}$ and chemically diverse with a minimal backbone consisting of a phosphatidylinositol group linked to a polysaccharide core (one glucosamine, four mannoses) modified with phosphoethanolamine (EtNP) (Fig. 1a). These complicated anchorages (Fig. S1a) place GPI-APs in lipid rafts and confer their unique regulatory properties in developmental and physiological processes 2-5. Notable GPI-APs include LY6K/TEX101 as key factors for fertilization and biomarkers for infertility ${ }^{8}$, glypicans/Gas1/RECK that modulate Hedgehog/Wnt/Notch signaling ${ }^{9,10}$, CD55/CD59 that inhibit complement cascade in innate immunity ${ }^{11}$, alkaline phosphatase as a leading biomarker for hepatic diseases and cancers ${ }^{12}$, and folate receptor 1 that mediates folate uptake and as an important cancer biomarker ${ }^{13}$. Disruption of the GPI-AP biosynthesis leads to embryonic lethality in animals ${ }^{14}$, while its biogenesis pathway in Trypanosoma brucei is a validated drug target for the fatal sleeping sickness ${ }^{15}$.

The committed step in GPI-AP biogenesis is catalyzed by the GPI transamidase (GPI-T), which cleaves the C-terminal signal peptide (CSP) of precursor protein and covalently links the EtNP moiety of GPI to the newly exposed carboxyl terminus of the so-called $\omega$-residue (Fig. 1a) ${ }^{1,2,16}$. GPI-T consists of at least five subunits (Fig. S1b), namely PIGK/Gpi8p, PIGT/Gpi16p, PIGU/Gab1p, PIGS/Gpi17p, GPAA1/Gaa1p in human/yeast, respectively (Fig. S2) ${ }^{2}$. For ease of description, we use the human 
nomenclature hereafter. PIGK and GPAA1 have been proposed to execute the peptide cleavage ${ }^{17,18}$ and GPI addition reactions ${ }^{19}$. PIGU and GPAA1 have been suggested to bind GPI ${ }^{16,20}$. PIGT disulfide-links with PIGK in some species ${ }^{21}$ and may play a structural role. The function of PIGS is less clear although it is essential for GPI-T activity ${ }^{22}$. All subunits are predicted to contain at least one transmembrane helix (TMH) except for PIGK in some species like T. brucei ${ }^{23}$. Aberrant activity of GPI-T has been recently implicated in multiple pathologies ${ }^{24-33}$ such as neurodevelopmental disorder with hypotonia and cerebellar atrophy, with or without seizures (NEDHCAS) and cancers. No experimental structural information exists to explain and clarify various and sometimes contradictory models for GPI-T's assembly $22,34,35$, membrane topology ${ }^{20,36}$, and subunit function ${ }^{16-20}$.

Here, we report the high-resolution cryo-EM structure of the human glycosylphosphatidylinositol transamidase (GPI-T). Combined with functional studies, our work represents an important step towards the mechanistic understanding of the GPI-AP biosynthesis and the pathophysiology associated with GPI-T mutations.

\section{Results}

\section{PIGT and PIGU form a platform for complex assembly}

To gain insights into the GPI anchoring process, we determined the cryo-EM structure of the transamidase complex at $2.53 \AA$ nominal resolution (Figs. S3a-c, Table S1). The high-quality map (Fig. S3d) sufficed $a b$ initio model building, with a total of 
2,393 residues (94.4\% completion), $3 \mathrm{~N}$-glycosylation sites, 4 disulfide bonds, and 22 lipid/detergent molecules. The overall GPI-T architecture assumes the shape of a canon (the luminal domain, mainly from PIGT/GPAA1/PIGS/PIGK) on a carriage (the transmembrane domain, TMD, mainly from GPAA1/PIGU) with an approximate dimension of $148 \AA$ by $141 \AA$ by $83 \AA$ (Fig. 1b). The TMD consisting of 24 TMHs is divided to contain a small entity with eight TMHs from GPAA1 and a large entity with a TMH core (TMH1-12 of PIGU) surrounded by four satellite TMHs, two from PIGS and one each from PIGT and PIGK.
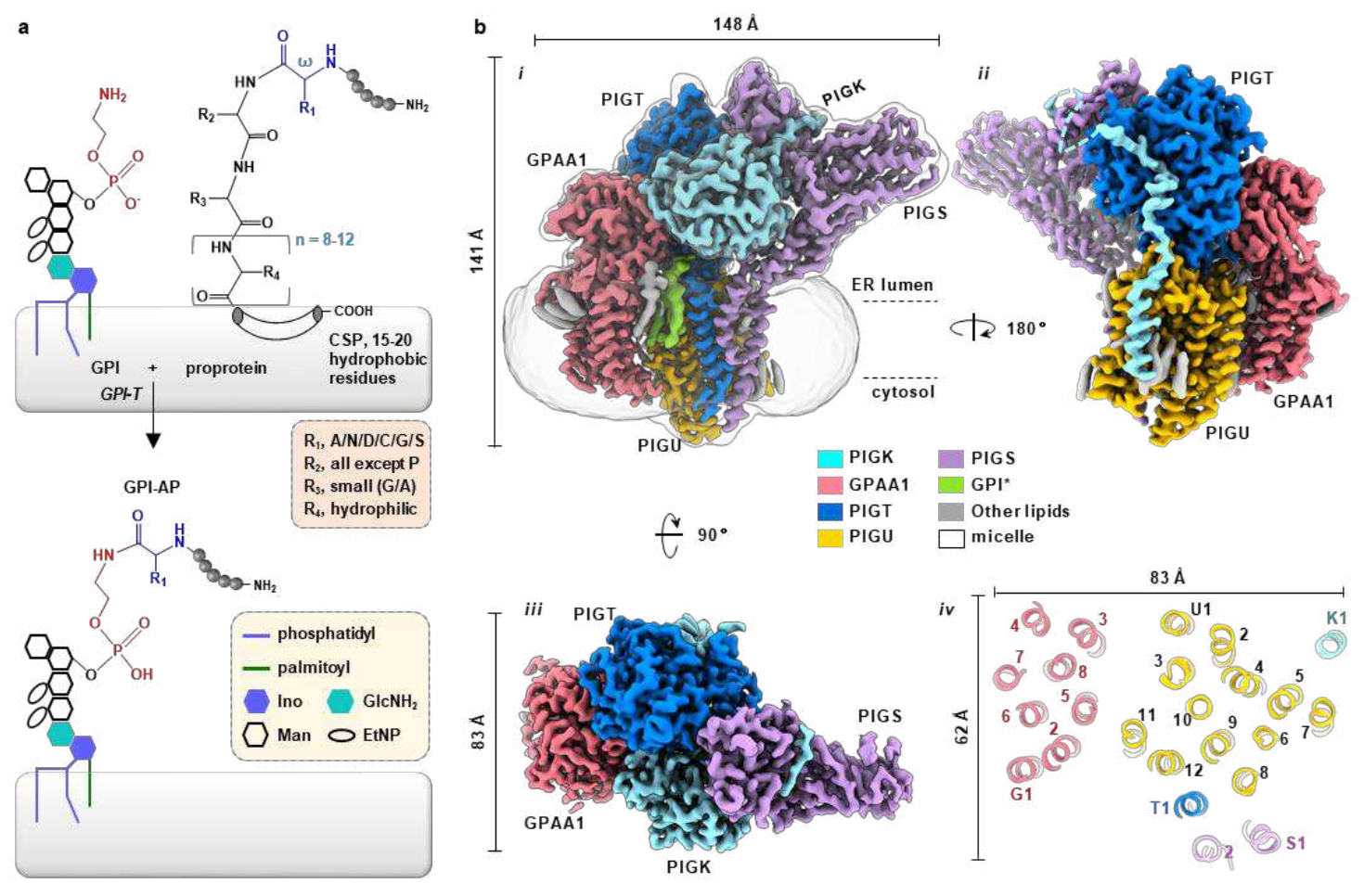

Fig. 1. Cryo-EM map of the human GPI-T. a GPI-T replaces the C-terminal signal peptide (CSP) of proproteins with GPI at the $\omega$ residue (blue) by a transamination reaction. Various parts are denoted in the dashed box. EtNP, ethanolamine; Man, mannose; Ino, inositol; GlnNH 2 , glucosamine. b Cryo-EM map (i-iii) and cross section view of the transmembrane domain $(\boldsymbol{i v})$. An asterisk indicates a partial GPI. Numbers 
in $\boldsymbol{i} v$ indicate TMHs and G/T/U/S/K refer to GPAA1 and PIGT/U/S/K, respectively.

Central to the assembly of the GPI-T complex is a platform formed by PIGT and PIGU (Fig. 2a). In the ER lumen, two lobes of PIGT (LbT1 and LbT2) pack together with skeleton features. Specifically, ten twisted antiparallel $\beta$-strands of LbT1 stack into a "half-rib" cage that was decorated with loops and short $\alpha$-helices for interaction with GPAA1/PIGK/PIGS, and LbT2 assumes a stable $\beta$-sandwich topology for interaction with GPAA1 and PIGU (Fig. 2b). As a result, $25.9 \%$ of its total surface area was buried by other subunits (Fig. 2a), conforming to its structural role as previously proposed ${ }^{22}$. In the ER membrane, PIGU recruits other subunits through an optimal geometry that maximizes interaction surfaces within the membrane and at the luminal surface. Specifically, its 12-TMHs are arranged into two centric rings (Figs. 2a, c). The outer ring consisting of TMH1/2/7/8/11/12 interacts with the transmembrane domains of all other four subunits alongside the membrane plane. The inner ring, however, contains six short TMHs that do not fully transverse the membrane, creating a hydrophobic void to strategically attract the five amphipathic helices (AH1-5) (Fig. 2c). In turn, these AHs expose several acidic residues and dipole moments (Fig. 2c) to create an overall negatively charged surface for electrostatic complementation with the corresponding PIGT surface (Fig. 2d). Thus, PIGU is arranged optimally as a docking base for PIGT and together with PIGT to buttress all other subunits. 

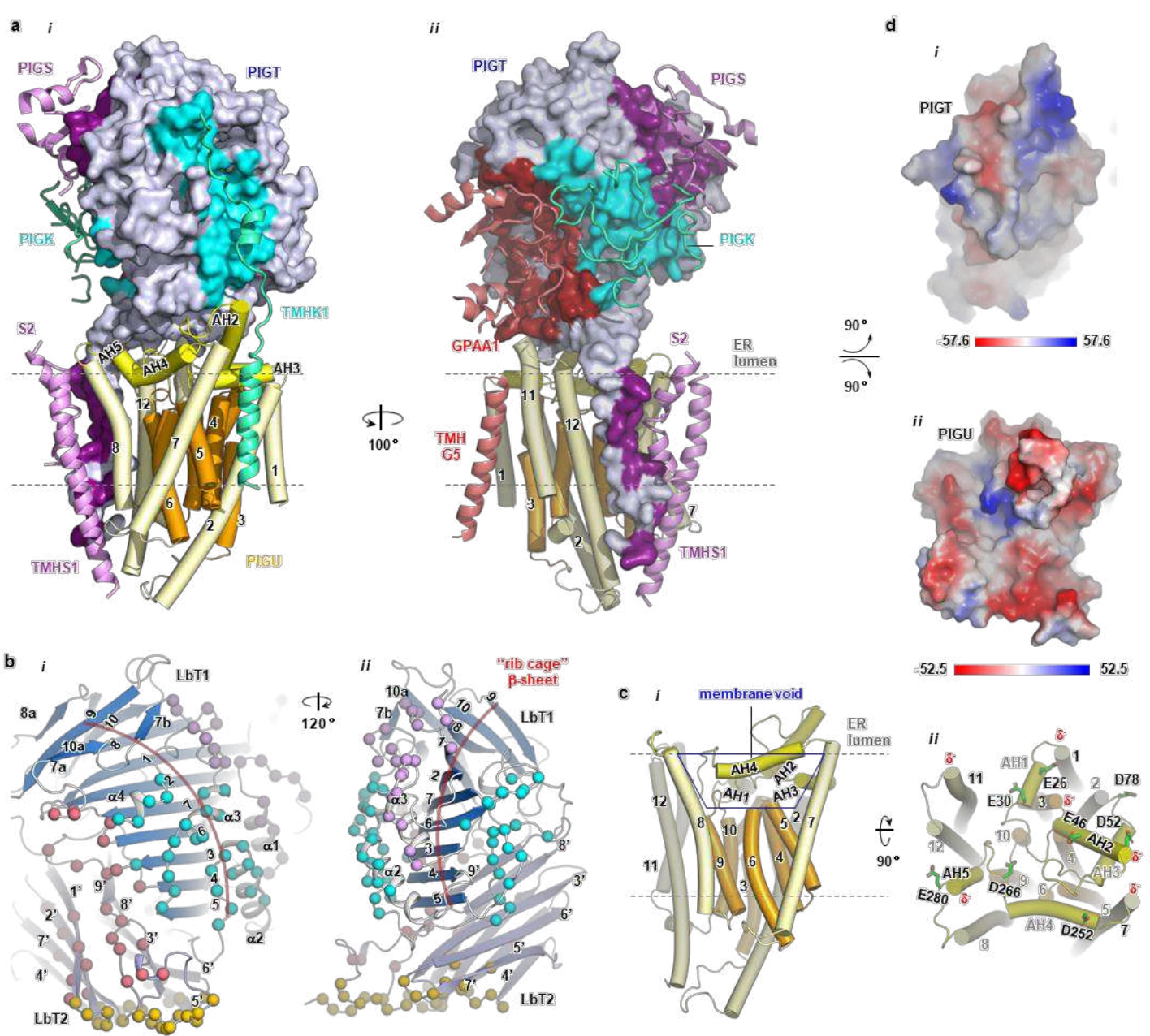

Fig. 2. PIGT and PIGU form a platform for complex assembly. a PIGT (surface

representations, blue) and PIGU (cylinder, yellow/orange) form a docking platform for 
short TMHs enclosed by a ring of TMHs (pale yellow). This arrangement creates a membrane void (blue trapezoid) to hold the five amphipathic helixes AH1-5 (yellow). The TMHs and AHs are so arranged such that the C-terminal ends of several $\alpha$-helices (labeled with black text) expose to the surface (ii). The resulting dipole moments $\left(\delta^{-}\right)$ and acidic residues make the surface electrostatically negative. d "Open-book" representation of the electrostatic potential molecular surface generated using the Adaptive Poisson-Boltzmann Solver module in PyMol.

\section{A complex assembled for catalytic purposes}

The consensually recognized catalytic subunit PIGK ${ }^{17,18}$ is secured onto the platform through multivalent interfaces. Its protease domain sits snugly in a three-sided cavity formed by the PIGT/PIGU platform as well as GPAA1 and PIGS approaching from opposite directions (Fig. 1b). In addition, PIGK exploits two grooves on the shamrock-like PIGS for interactions, with the globular luminal domain complementing the concave Groove1 surface and its "belt"-like loop entangling onto the Groove2 (Fig. 3a). This configuration buries a total of $2341.9 \AA^{2}$ surface area and holds PIGK tightly via 18 hydrogen bonds, 6 salt bridges, one cation- $\pi$ interaction, and several hydrophobic interactions (Fig. 3b). Further, the C-terminal portion of PIGK travels down along a shallow surface of PIGT's backside and buckles its terminal helix into the membrane to interact with TMH5/7 of PIGU (Figs. 1b, 2a). Finally, an inter-subunit disulfide bond between PIGK C92 and PIGT C182, which has been reported previously ${ }^{21}$ and 
visualized in this study, nails PIGK onto PIGT (Figs. 3c, d). These interactions place PIGK at a "mid-air" position (relative to the membrane "ground") with its catalytic dyad (C206-H164) ${ }^{17}$ measuring $\sim 22 \AA$ from the membrane (Fig. 3c). Because the distance approximates the hydrophilic "stem" of both GPI and proprotein substrates that are presumably rooted in the membrane (Fig. 1a), this topological arrangement may confer substrate specificity.

a

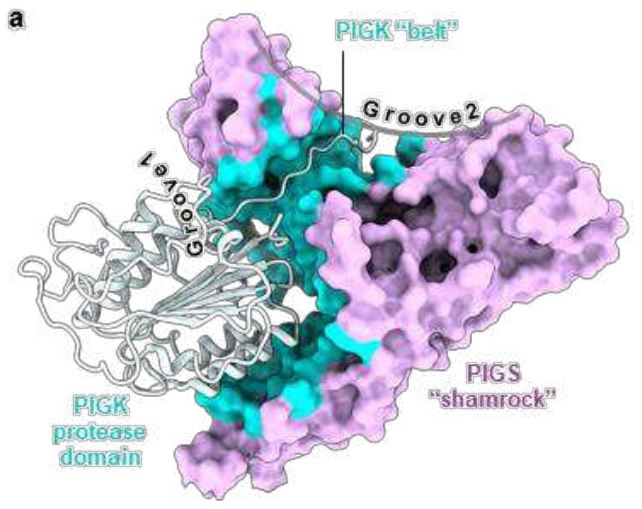

b $\quad$ i

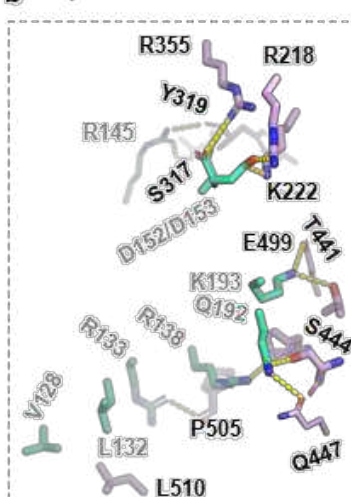

\&510

Groove 1 c
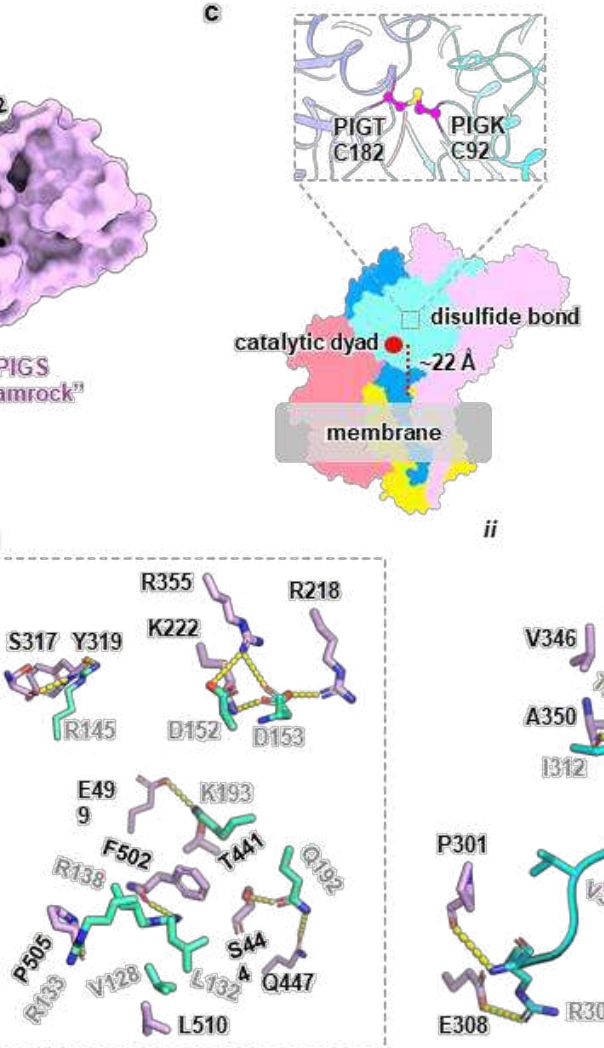

d

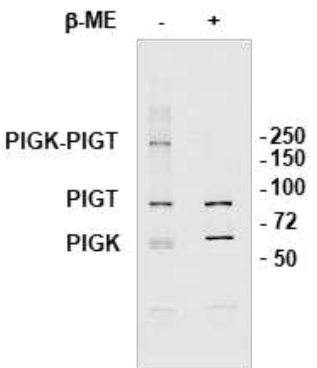

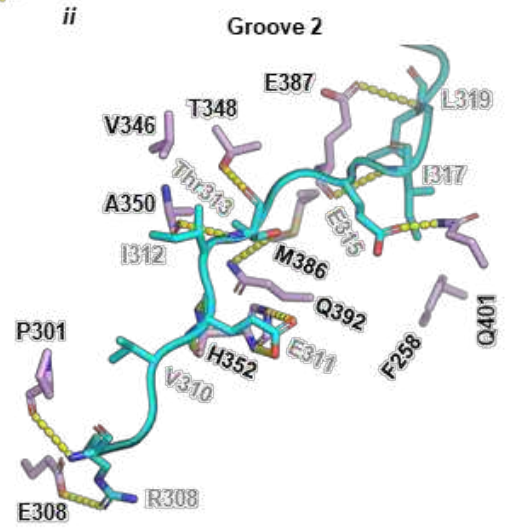

Fig. 3. PIGS and PIGT place PIGK and its catalytic dyad in a position suitable for

catalysis. a PIGS (surface, pink) holds PIGK (ribbon) by embracing the protease domain using one of the shamrock grooves and hosting the loop region in another groove. The buried surface is colored cyan. b Detailed interactions between PIGK and 
PIGS at Groove 1 (i) and Groove 2 (ii). PIGK residues (cyan) are indicated with grey texts and PIGS residues (light purple) are labeled with black texts. Dashed lines (yellow) indicate distances within 3.6 A. c An inter-subunit disulfide bond (PIGT C182 / PIGK C92) (magenta) nails PIGK (cyan) onto the PIGT (blue). This and the other interactions fix PIGK in a position such that the catalytic dyad (red dot) is $\sim 22 \AA$ "above" the membrane interface (grey shading). This geometry would suit interactions with GPI which is expected to insert into the membrane by the phosphatidyl moiety with its hydrophilic glycan chains (measuring $\sim 25 \AA$ in the extended form) stemming from the membrane to meet with the catalytic dyad. d Verification of the PIGK-PIGT disulfide bond. SDS-PAGE in-gel fluorescence shows a high molecular-weight band (PIGKPIGT) in the absence of, but not in the presence of, the reducing agent $\beta$ mercaptoethanol ( $\beta-\mathrm{ME})$ at the cost of the individual PIGT/PIGK bands. Other subunits were also co-transfected but were invisible owing to their lack of the TGP-tag ${ }^{37}$. Molecular markers are labeled on the right. 


\section{A legumain-like active site}

PIGK belongs to the $\mathrm{C} 13$ cysteine protease family including legumains and caspases ${ }^{17,18}$. In the GPI-T structure, PIGK features a central 6-stranded $\beta$-sheet with $3 \alpha$-helices on either side (Figs. S4a, b) and structurally resembles legumains $(\mathrm{C} \alpha-$ RMSD of $2.3 \AA$ ) (Fig. S4d) ${ }^{38}$. The catalytic dyad, the trivalent oxyanion hole, and the residues in the substrate-binding pockets $\mathrm{S} 1 / \mathrm{S} 1^{\prime} / \mathrm{S} 2$ ' are mostly conserved and superimposable (Figs. 4a, S4d, e).
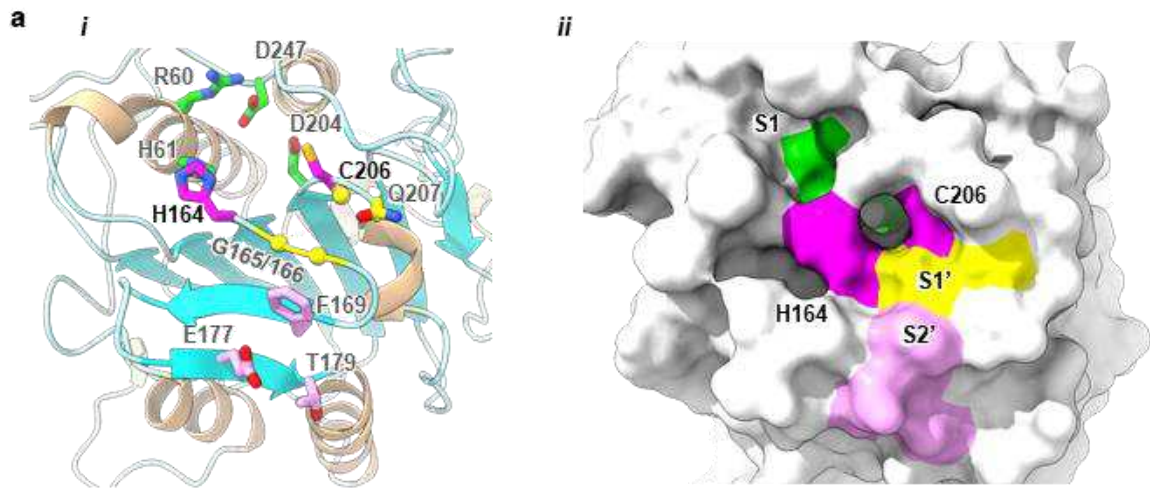

b

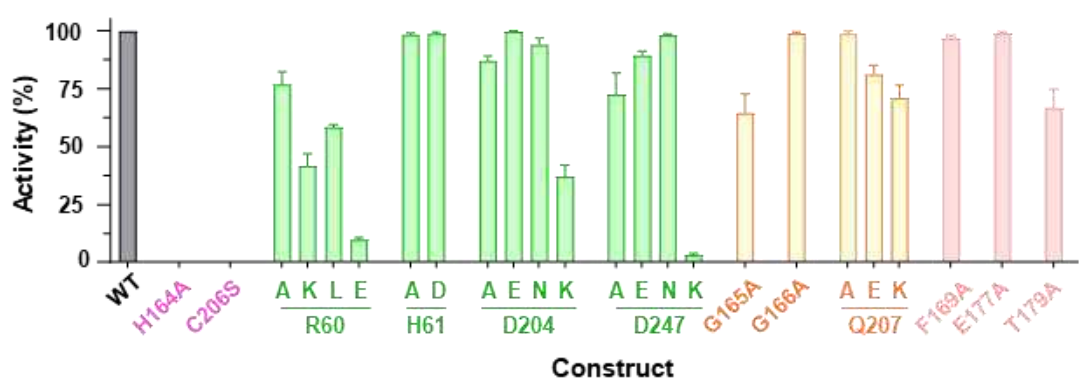

Fig. 4. Structural and functional resemblance of the PIGK active site to that of legumains. a Cartoon $(i)$ and surface (ii) representation of the PIGK protease domain with the catalytic dyad (magenta), S1 (green), S1' (yellow), and S2' (pink) residues shown as sticks $(\boldsymbol{i})$ or highlighted in colors $(\boldsymbol{i i}) . \mathrm{S} 1 / \mathrm{S} 1$ '/S2' sites are superposed from 
legumain structures ${ }^{39,40}$. b Functional assay of the active site mutants. Apparent activity (\% of wild-type, WT) was measured by immune staining of a reporter GPI-AP (CD59) on the surface of PIGK-KO cells transfected with the indicated mutants of the catalytic dyad and $\mathrm{S} 1 / \mathrm{S} 1^{\prime} / \mathrm{S} 2{ }^{\prime}$ residues. Cells expressing PIGK fused with a thermostable green fluorescence protein (TGP) ${ }^{37}$ were gated, and the surface staining of CD59 was analyzed. Data represent mean \pm s.e.m. from three independent experiments (Supplementary Data 1).

To ascertain the functional importance of S1/S1'/S2' residues, we used a cell-based GPI-AP reporter assay ${ }^{20}$ to probe the apparent activity of the mutants. In the cell-based assay, the surface display of the reporter CD59 was monitored by flow cytometry in GPI-T knockout HEK293 cells upon ectopic expression of mutants. As a control, mutations of the previously identified catalytic dyad ${ }^{17}$ (H164A or C206S) abolished GPI-T activity (Fig. 4b).

Substituting R60 at the S1 site with glutamate almost diminished GPI-T activity (9.8\% relative to the wild-type (WT) and same hereafter), and R60A, R60L, or even the conservative R60K mutation reduced the activity by 20\%-60\% (Figs. 4b, S5a, Supplementary Data 1), suggesting that both the shape and the positive charge of R60 were important for the integrity of S1. The negative charge on D204 or D247 was not essential (D204N, 94.2\%; D247N, 98.2\%) but a charge reversion was detrimental (D204K, 37.3\%; D247K, 3.2\%). In contrast, H61 tolerated mutation to alanine or even 
aspartate (H61A, 98.5\%; H61D, 98.7\%) (Fig. 4b), suggesting a less important role. The mutagenesis results also indicate the functional importance of three residues in the corresponding S1' (G165A, 64.5\%; Q207E, 81.2\%, Q207K, 71.2\%) and S2' site (T179A, 66.7\%) (Fig. 4b) ${ }^{39,40}$. Taken together, PIGK may use a similar mechanism as legumains for peptide cleavage and even substrate recognition.

\section{GPI-T contains two protease-like domains}

Both GPAA1 and PIGS possess a protease-like domain in our GPI-T structure. GPAA1 assumes a portico-shape with eight TMHs, four AHs, and a soluble domain (Fig. 5a). Whilst the transmembrane domain shares no recognizable structural homology with known folds, the soluble domain is similarly arranged as the $\mathrm{Zn}^{2+}$ protease AM-1 ${ }^{41}$ (PDB ID 2EK8, C $\alpha$ RMSD of $3.2 \AA$ ) (Fig. 5b). However, a closer inspection shows the corresponding GPAA1 domain lacks a characteristic catalytic zinc-binding motif that consists of glutamates, aspartates, and histidines (Fig. $\mathbf{5 c}$ ), and mutations of possible Zn-binding motif candidates in the vicinity (D153A, E186A, H187A, D188A, E226A, H303A) (Fig. 5d) had no noticeable effect on GPI-T activity in cells (Fig. 5e). Likewise, a portion of PIGS (resi. 222-398) folds similarly to a metzincin-type protease AmzA (C $\alpha$ RMSD of $3.6 \AA)^{42}$ (Fig. S6a). However, unlike AmzA, PIGS lacks elements for a metzincin active site that contains a conserved methionine near a zinc-binding tri-histidine motif. Further, a cysteine-based zinc-finger in AmzA was compositionally impossible in PIGS (Figs. S6b, S6c). Thus, we envisage 

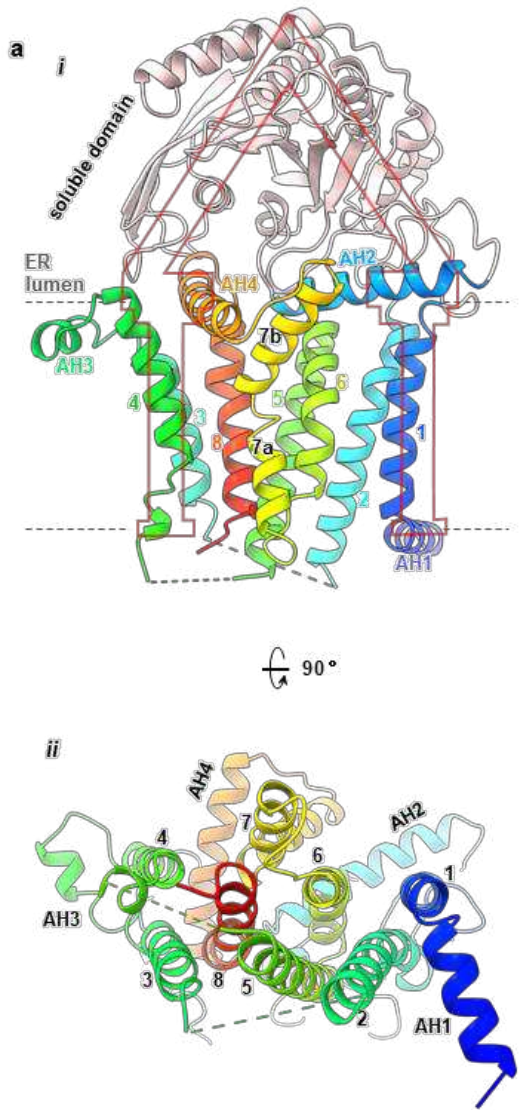
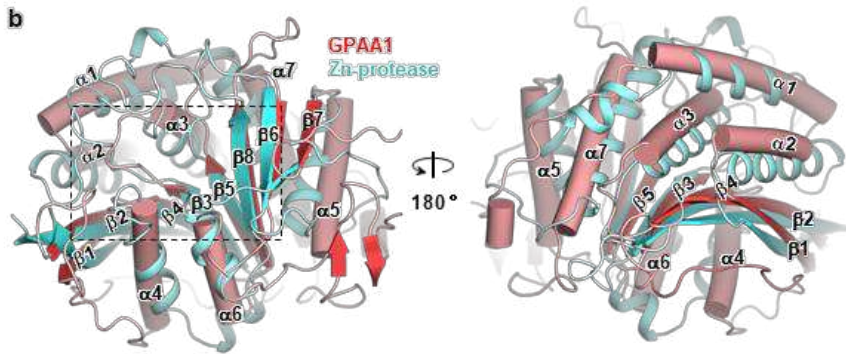

c
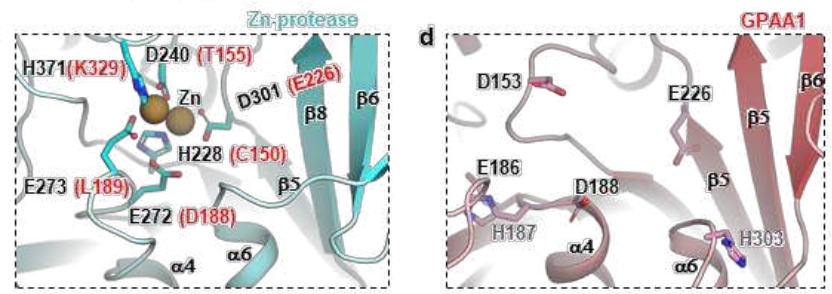

e
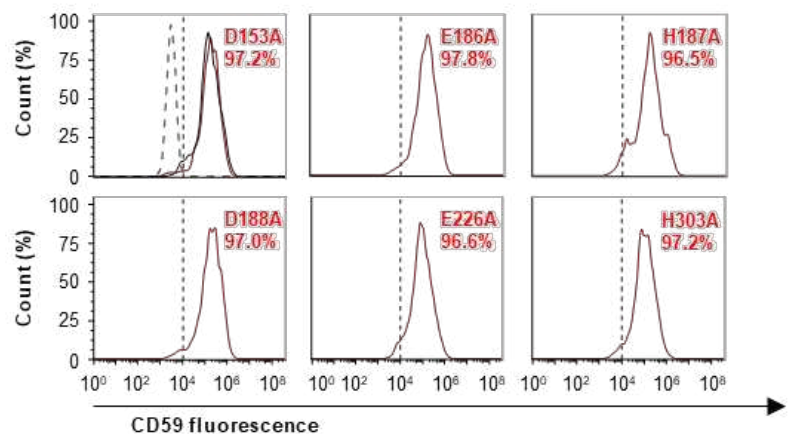

Fig. 5. Structural and functional characterization of GPAA1 reveal a protease-like

domain. a Side (i) and normal (ii) view of GPAA1. Numbers indicate TMHs and AH13 label the three amphipathic helices. b The soluble domain of GPAA1 (red/pink, cylinder) is structurally similar to a Zn-protease AM-1 (cyan, cartoon, PDB ID 2EK8) with a $Z$-score of 20.6 and C $\alpha$ RMSD of $3.2 \AA$ (from a DALI search) ${ }^{41}$. GPAA1 is shown as red/pink cylinder representations and the $\mathrm{Zn}$-protease is shown as cyan cartoon representations. $\mathbf{c}$ The Zn-binding site of AM-1 (expanded view of the boxed region in b) consists of two each of aspartate, glutamate, and histidine residues that are 
not fully conserved in GPAA1 (in brackets). d Arrangement of GPAA1 aspartate/glutamate/histidine $(\mathrm{D} / \mathrm{E} / \mathrm{H})$ residues in the region corresponding to the $\mathrm{Zn}$ binding site in AM-1. Despite having the same composition, these residues are unlikely to form a Zn-binding site because of different spatial arrangements. e Mutation of the $\mathrm{D} / \mathrm{E} / \mathrm{H}$ residues in $\mathbf{d}$ did not reduce CD59-staining in the flow cytometry assay. The function of wild-type GPAA1 and mutants were assessed using FACS analysis which monitors the surface expression of the GPI-AP reporter (CD59) in GPAA1 KO cells transfected with appropriate plasmids. Cells were gated by TGP fluorescence ${ }^{37}$ for GPAA1 expression and analyzed for CD59 staining. The dotted line (grey) indicates the CD59-staining background level from cells expressing an unrelated TGP-tagged membrane protein (negative control). Solid lines indicate staining of cells transfected with the wild-type (black) or mutant genes. A vertical dash line marks threshold (CD59gating) determined from the negative control.

\section{Identification of the GPI-binding site}

The lipid substrate GPI is, as aforementioned, expected to be anchored in the membrane via its phosphatidyl group within $\sim 25 \AA$ of the catalytic dyad based on the approximate length of the glycans between the reactive EtNP3 and the acyl chains. Intriguingly, densities that fit an almost complete GPI core (palmitoylated phosphatidylinositol, glucosylamine, and EtNP-modified mannose1) (Fig. 6a) are observed in a cavity jointly formed by TMH11/12 of PIGU, TMH2 of GPAA1, and the 
sole TMH of PIGT (Fig. 6b) directly "underneath" the catalytic dyad. Although the precise orientation of the EtNP1 moiety is ambiguous at the current resolution, the density for the characteristic tri-acyl chain, the inositol-glucosylamine-mannose glycan core is well-resolved. In the model, the acyl chains of GPI glue to the transmembrane region mostly of PIGU, and the head group forms three strong hydrogen bonds with PIGT D521/S523 and several weak ones with PIGU N383/N385, and GPAA1 H354/Q355 (Fig. 6c). This composite cavity is additionally filled with a density that fits nicely for a digitonin molecule which also contains a polysaccharide chain like GPI (Fig. S7a). Further, several evolutionarily conserved residues (PIGK F55/D174/S175, PIGT Y456/D459/P460/N461/D521/F522/S523/M524/N527) (Figs. S7c, d) delineate a surface-exposed patch stretching from the membrane interface to the catalytic dyad (Fig. 6b). Notably, this motif is rich in acidic/aromatic residues that are capable of forming H-bonds/"greasy slides" frequently found in protein-sugar interactions ${ }^{44}$. Making topological sense, the cavity is oriented in a way it would fit a proprotein substrate with the hydrophobic portion of CSP in the membrane while the hydrophilic portion would span the $\sim 22 \AA$ space to reach the active site (Fig. 6b). 

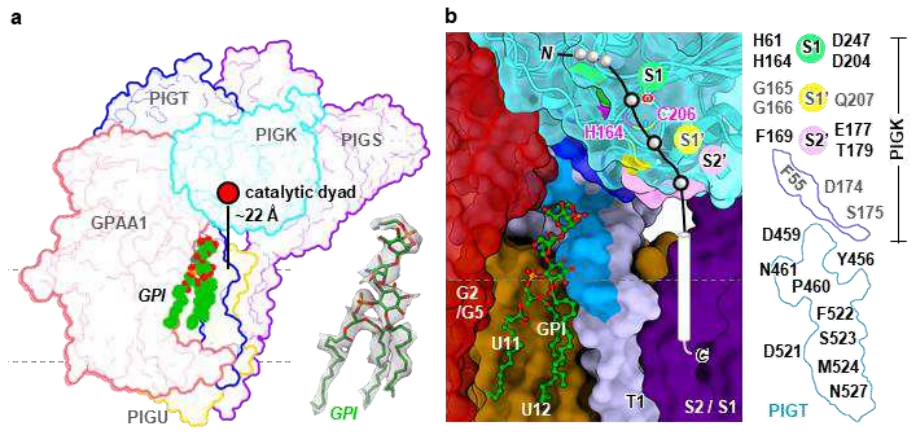

d

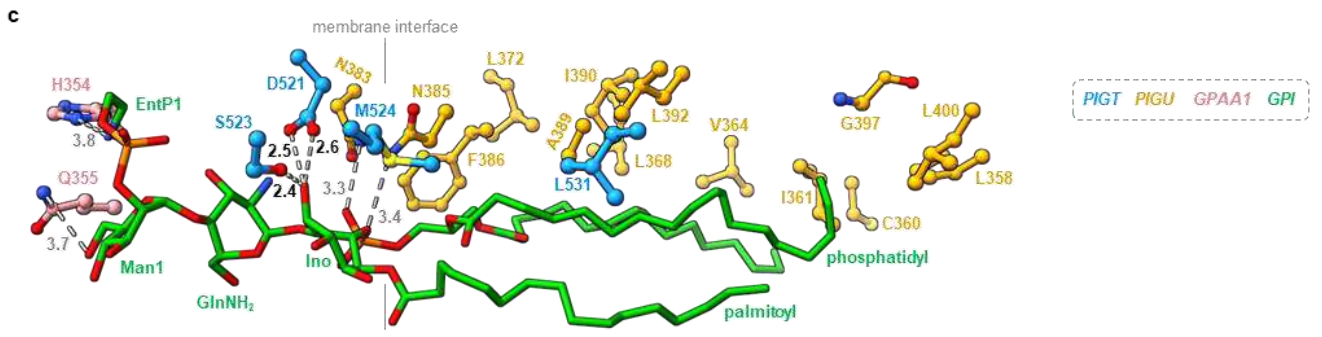

Fig. 6. Characterization of a composite GPI-binding site. a Cryo-EM density that

fills a nearly complete GPI was observed in the membrane cavity "underneath" the catalytic dyad. b Expanded view of the composite site encompassed by the indicated TMHs from GPAA1/PIGU/PIGT/PIGS. Evolutionarily conserved residues (Figs. S7c, d) in the vicinity are colored marine (PIGT) and blue (PIGK). Subunits are shown as surfaces except that PIGK was additionally shown as ribbon representations with the catalytic dyad and S1/S1'/S2' residues highlighted in indicated colors. c Interaction between the partial GPI (green) and GPI-T (colored-coded as indicated). Distances $(\AA)$ are either indicated by numbers (black/grey for strong/weak H-bonds) for H-bonding interactions or omitted for hydrophobic interactions (within $5 \AA$ of GPI). A vertical line marks the membrane boundary. d Apparent activity of GPI-T mutants relative to the wild-type (WT). GPI-T KO cells were gated by TGP-fluorescence ${ }^{37}$ for subunit expression and analyzed for surface staining of the reporter GPI-AP (CD59) by flow cytometry. Data represent mean \pm s.e.m. from three independent experiments (Supplementary Data 1). 
1) targeting 10 residues in the conserved patch and the hydrogen-bonding network involved in GPI-binding. Three residues, two from PIGT and one from GPAA1, responded appreciably to mutagenesis. D521 formed two strong H-bonds with GPI (Fig. 6c). Consistently, substituting it with an alanine decreased the apparent activity to $28.9 \%$, and introducing intended steric clashes by a leucine reduced the activity to 18.8\%. The H-bond by PIGT S523 was seemingly non-essential (S523A, 98.4\%), but mutating to bulky residues reduced apparent activity (S523F, 76.5\%; S523W, 76.8\%) (Fig. 6d) presumably by steric hindrance. Further, a double mutant (D521L/S523F) almost abolished activity (6.2\%, Fig. 6d). GPAA1 H354 formed a weak H-bond with GPI. Although the alanine mutation did not change the apparent activity, substitution with phenylalanine which has a similar volume with histidine reduced activity by $\sim 25 \%$ (Fig. 6d). The lack of substantial change on GPI-T activity for individual mutants of other residues (Fig. S7b) might be explained by the abundance of weak multivalent interactions with GPI.

\section{Structural interpretation of pathogenic mutations}

Our structure provides a framework to propose possible mechanisms for genetic neurodevelopmental disorders such as NEDHCAS associated with GPI-T mutations and reduced surface expression of various GPI-APs ${ }^{24-33}$. Mapping these mutations onto GPI-T showed a pattern with over half of the mutations (12 out of 22, PIGK 
S51L/A389P, and PIGU N383K) clustering near the catalytic dyad and the GPI-binding site (Figs. 7, S8a). Among the rest of the ten mutations, eight are located at the intersubunit interfaces (PIGT T183P/E184K/G360V /G366W/R488W, PIGS E308G, GPAA1 W176S, and PIGU I70K) (Figs. 7, S8b) and hence may disrupt the integrity of the complex. PIGS L34P occurs in the first transmembrane helix and may cause folding issues by main chain distortion. PIGK Y160S and GPAA1 W176S may also promote misfolding by introducing the small hydrophilic serine residue into a hydrophobic microenvironment (Figs. S8c, d).
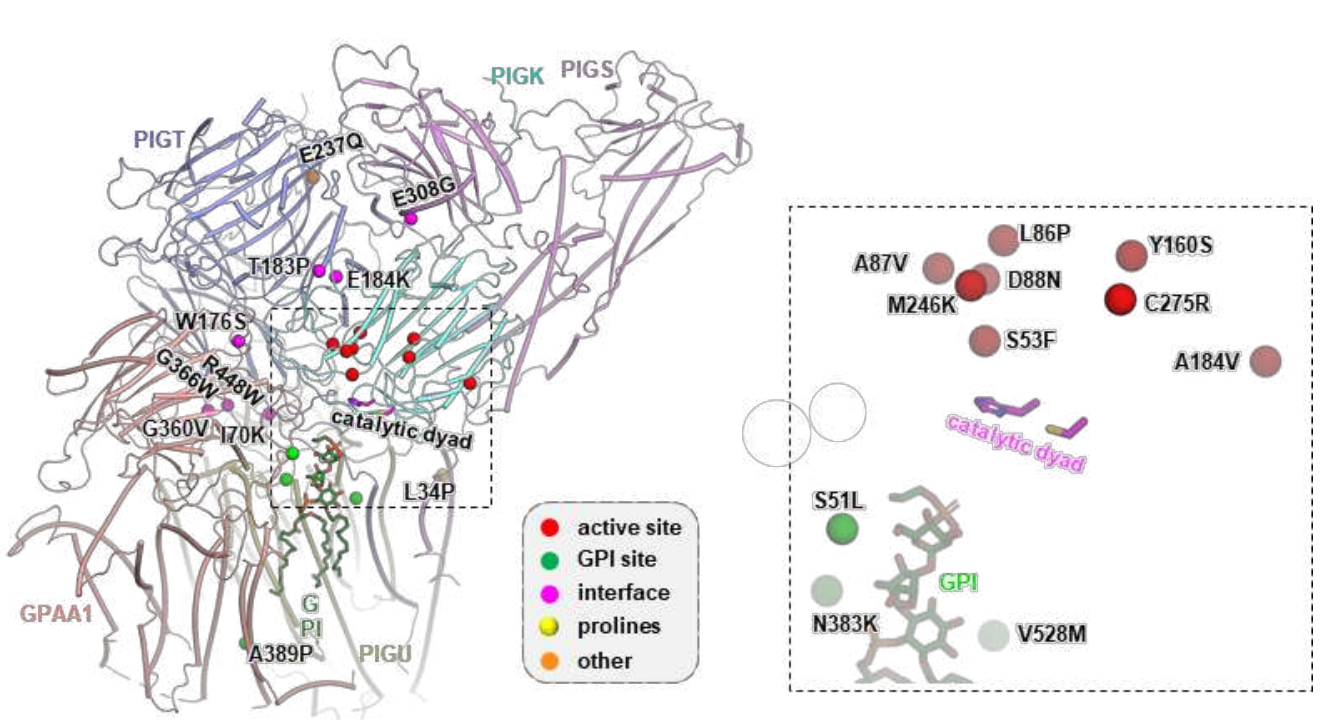

Fig. 7. Distribution of genetic mutations on GPI-T. Mapping the disease mutations ( $\mathrm{C} \alpha$ spheres, color-coded by the categories in the grey box) onto the human GPI-T structure (cylinder representation). The mutations include PIGK S53F/L86P/A87V/ D88N/Y160S/A184V/M246K/C275R that cause a neurodevelopmental syndrome with hypotonia, cerebellar atrophy, and epilepsy ${ }^{24}$, PIGT T183P that causes an intellectual disability syndrome ${ }^{27}$, PIGT E184K/G360V/R448W that are related to the Multiple 
Congenital Anomalies-Hypotonia Seizures Syndrome $3{ }^{28,31,32}$, PIGT E237Q/V528M that cause developmental disorders characterizing learning disability, epilepsy, microcephaly, congenital malformations and mild dysmorphic features ${ }^{29}$, PIGT G366W that is found in patients with epileptic apanea and multiple congenital anomalies, severe intellectual disability, and seizures ${ }^{30}$, PIGS L34P/E308G that are related to a neurological syndrome ranging from fetal akinesia to epileptic encephalopathy ${ }^{26}$, PIGU I70K/N383K that are found in patients with severe intellectual disability, epilepsy, and brain anomalies ${ }^{25}$, and GPAA1 S51L/W176S/A389P that are also related to developmental disorders featuring global developmental delay, epilepsy, cerebellar atrophy, and osteopenia ${ }^{33}$.

\section{Discussion}

The yeast GPI-T has been reported to exist in dimer based on native PAGE results 45. Although we have not observed dimer particles for the human GPI-T in this study, we note the report of an increasing number of detergent-specific weak oligomers of membrane proteins in the literature ${ }^{46-49}$. Future investigations using less disruptive detergents such as steroid glycosides for extraction may be considered. Such detergent screening experiments, and in fact enzyme kinetics studies, would greatly benefit from a robust test-tube biochemical assay with authentic substrates which is yet to be developed.

Although the substrate-binding and catalytic mechanisms are yet to be revealed by structural studies of GPI-T with the peptide substrate, the structural comparison to 
legumains offers mechanistic implications. In legumains, the trivalent oxyanion pocket ${ }^{40}$ (backbone amide nitrogen of $\mathrm{C} 189, \mathrm{G} 149$, and $\mathrm{N} \delta 1$ of the catalytic residue H148) is proposed to polarize the carbonyl oxygen of the P1 residue in the substrate. This increases the electrophilicity of the carbonyl carbon, allowing the catalytic C189-S $\gamma$ to deprotonate and to launch the nucleophilic attack that proceeds to peptide cleavage. In PIGK, the corresponding PIGK residues are the similarly positioned C206, G165, and H164 (Fig. S4d, e), indicating an analogy in the carbonyl activation process. In legumains and caspases, the selectivity of the substrate P1 residue is conferred mainly by the S1 pocket, and the characteristic zwitterionic S1 site (two positively charged residues and two negatively charged residues) of legumains is believed to be suited for its asparagine specificity. In line with this, legumains are unable to process substrates with an aspartate unless low-pH conditions are used to protonate the P1 residue ${ }^{40}$. Interestingly, despite being capable of processing substrates with various $\omega$-residues (analogous to P1) including aspartate, the corresponding site in PIGK has virtually the same composition as the $\mathrm{S} 1$ of legumains with the exception of a conservative change (PIGK D204 versus legumain E187) (Figs. 4a, S4e). Whether and how this site confers selectivity to proprotein substrates remains to be investigated structurally and biochemically.

In addition to the protease activity, a ligation reaction to conjugate the GPI-peptide amide, presumably also by PIGK, is required for GPI anchoring. In legumains ${ }^{50}$, the ligase activity involves a recently discovered mechanism where a succinimide residue converted from D147 provides energy. Despite its overall similarity with legumains, 
PIGK contains a glycine (G163) as a counterpart to the legumain D147 (Fig. S4a), thus making the succinimide-mediated ligation less likely in PIGK. Instead, PIGK may use a ping-pong mechanism similar to that of the sortase transamidation ${ }^{51}$ : the catalytic dyad acts on the pro-protein, forming a semi-stable thioacyl intermediate that links the $\omega$ residue and $\mathrm{C} 206$. The incoming amine group on EtNP then attacks this intermediate, producing GPI-AP and freeing PIGK.

GPAA1 has been proposed previously to be a catalytic subunit owing to its similar arrangement of secondary structure elements to that of zinc proteases ${ }^{19}$. We verify the structural similarity but clarify that GPAA1 lacks the Zn-binding motif and hence is unlikely a catalytic subunit (Figs. 5b-e). Furthermore, the region corresponding to the AM-1 Zn-binding site is remote ( $50 \AA)$ from the catalytic dyad in our structure (Fig. S9).

Both GPAA1 and PIGU have been proposed to be involved in GPI-binding. The functional part was narrowed down to the last TMH for GPAA $1{ }^{20}$ which is wrapped by the rest of GPAA1 TMHs (Fig. 5a). Because this site is $\sim 20 \AA$ away from the composite GPI-binding site (Fig. S9), how GPAA1 TMH8 is involved in GPI-binding is currently unclear. Similarly, residues F274/W275, located in the AH5 of PIGU (Fig. S2c), are proposed to be crucial for GPI-binding ${ }^{16}$. Although the functional importance of the two residues is verified in this study (Supplementary Data 1), they are also distal ( 10 $\AA$ ) to the composite GPI-binding site (Fig. S9). It is possible that mutations in these regions compromise GPI-binding by allosteric effects. 
The density for a partial GPI molecule in the structure provides valuable

information for an overview of the active site, but the detailed mechanism, for example, the requirement of the EtNP3 for GPI-T reaction, remains unknown because of the lack of reliable density beyond Man1. In addition to improving resolution, designing mutants to covalently trap GPI or GPI-APs to the active site should help reveal the complete picture of substrate-binding.

Finally, it is interesting to note the implications of the glycan-containing digitonin molecule proximal to the composite GPI-binding site. Because lipids mostly diffuse two-dimensionally in the membrane plane, GPI-T may use the cavity as a "hamster" pocket to stuff GPI. By doing so, the relatively low diffusion rate of lipids may be compensated by the locally high concentration of substrates in a reaction.

\section{Conclusions}

Our study on the human GPI-T complex provides structural evidence of an assembly that suits its catalytic purpose. The subunits PIGU/T/S and GPAA1 jointly hold PIGK in a way the space between the catalytic dyad in PIGK and the membrane interface presumably may act as a molecular ruler to restrict proprotein and GPI substrates. Combined with rational mutagenesis, the structural reveals a composite GPIbinding site where a juxtamembrane portion of PIGT is unexpectedly found to contribute most for the interactions. Consistent with the structural interpretations, most disease mutations occur near the catalytic site, the GPI-binding site, and at the subunit 
interfaces. Our work also formulates feasible working models for the GPI anchoring process and provides a high-resolution framework to inspire the design of biochemical and biophysical experiments to probe catalytic mechanisms in detail.

\section{ACKNOWLEDGEMENTS:}

Cryo-EM data were collected at SKLB West China Cryo-EM Center and processed on Duyu High Performance Computing Center in Sichuan University. We thank the staff members of the Center of Cryo-EM of Fudan University and the CryoEM Center at National Facility for Protein Science in Shanghai for technical support and assistance. We thank Drs. Feilong Meng, Liu Daisy Liu, and Hai Jiang and Mr. Yin Chen for guidance on CRISPR-Cas9. This work has been supported by the National Natural Science Foundation of China (82151215, 31870726, D.L; 32171194, Q.Q.; 82041016, 32070049, Z.S.), the Strategic Priority Research Program of CAS (XDB37020204), CAS Facility-based Open Research Program (2017, D.L.), Science and Technology Commission of Shanghai Municipality (20ZR1466700, D.L.), Ministry of Science and Technology of China (2021YFA1301900, Z.S.), and the startup funds from Shanghai Stomatological Hospital \& School of Stomatology, Fudan University (Q.Q.).

\section{AUTHOR CONTRIBUTIONS:}

Y.X. established purification protocol. T.L. and Y.X. purified the complex. Y.X. 
constructed KO cells and performed functional assays. Y.L., Y.C., and Z.Z. prepared and screened cryo-EM grids. G.J. collected cryo-EM data under the supervision of Z.S.. Z.Z. and G.J. processed cryo-EM data and produced the final map. J.B. helped with molecular cloning. D.L. initiated the project. D.L. and Q.Q. wrote the manuscript with input from Y.L., T.L., Y.X., Z.Z., and Z.S.

\section{COMPETING INTERESTS:}

The authors declare no competing interests.

\section{Methods}

\section{Molecular cloning}

The genes encoding the human GPI-T subunits GPAA1 (Genbank ID NP_003792.1), PIGK (NP_005473.1), PIGS (NP_149975.1), and PIGT (NP_057021.2) were PCR-amplified from cDNA clones provided by the authors' institute. The gene encoding the human PIGU (NP_536724.1) was PCR amplified using overlapping oligonucleotides. The PCR products were Gibson assembled ${ }^{52}$ into various versions of the $\mathrm{pBTSG}^{37}$ vector which were modified to carry the following tag sequences at the

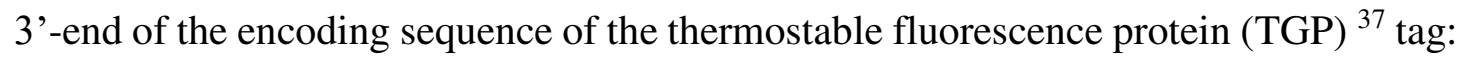
GPAA1, 2×Flag; PIGK, Strep; PIGS, Myc; PIGT, 9×His; PIGU, hemagglutinin (HA). For disulfide cross-linking between PIGT and PIGK, the DNA fragment encoding TGP 
was removed in the constructs of GPAA1/PIGS/PIGU to avoid background in in-gel fluorescence. The constructs were verified by DNA sequencing.

Mutations were made using standard PCR-based site-directed mutagenesis. DNA sequences were verified by Sanger sequencing.

The TGP-tags are located in the cytosol side of the GPI-T, opposite to its large luminal domain. Flow cytometry (below) showed that the TGP-tagged GPI-T was functional because the co-transfection of all five TGP-tagged subunits in a cell line with all GPI-T subunits disrupted (see below) restored cell surface expression of the GPI-AP reporter CD59.

\section{Generation of GPI-T knock-out (KO) cell lines}

To generate cells lines with defective individual subunits, the endogenous genes encoding the five GPI-T subunits were separately disrupted by CRISPR-Cas9 editing using two or three pairs of sgRNA oligos that are designed using the online server (http://cistrome.org/SSC/) ${ }^{53}$ with the following sequences (forward / reverse pairs):

5'- CACCGTGTGGGGCTGCTGCTGGCAC-3' / 5'- AAACGTGCCAGCAGCAG CCCCACAC-3' and 5' - CACCGCAGGAGCAAGAAGCCGACAG-3' / 5' - AAAC CTGTCGGCTTCTTGCTCCTGC-3' for GPAA1; 5' - CACCGAATTACCAACATA GAACTCG -3' / 5' - AAACCGAGTTCTATGTTGGTAATTC -3', 5' - CACCGTTC ATATTAGTTTGGCTAGC -3'/ 5' - AAACGCTAGCCAAACTAATATGAAC -3' and 5'- CACCGGCTCTAGCTAGTAGTCAAGT -3' / 5' - AAACACTTGACTACTAGCT 
AGAGCC -3' for PIGK; 5' - CACCGTGAGCCTCAGGAACAAGCGG -3', 5' - AAA CCCGCTTGTTCCTGAGGCTCAC -3' and 5'- CACCGAGTGGAGCGCTGAGAA GAGG-3' / 5' - AAACCCTCTTCTCAGCGCTCCACTC-3' for PIGS; 5'-CACCGC GGTGCAGACCACCTCCCG -3' / 5' - AAACCGGGAGGTGGTCTGCACCGC-3' and 5'- CACCGCACCATCACCTCCAAGGGCA-3' / 5'- AAACTGCCCTTGGA GGTGATGGTGC-3' for PIGT; 5'- CACCGAGTCCTGGATTGCAAAATAC-3' / 5'AAACGTATTTTGCAATCCAGGACTC -3', 5'- CACCGCCTAATTGACTATGCT GAAT-3' / 5' - AAACATTCAGCATAGTCAATTAGGC -3' and 5'- CACCGTCTTT GGGTAGTCAAAGTGA-3' / 5'- AAACTCACTTTGACTACCCAAAGAC-3’ for PIGU.

The oligos were designed to have sticky ends that are compatible with the Type IIs restriction enzyme BbsI (Cat. R3539S, NEB) after annealing. The sgRNA oligo pairs, dissolved in a buffer containing $0.2 \mathrm{M} \mathrm{NaCl}, 0.1 \mathrm{mM}$ EDTA, and $10 \mathrm{mM}$ Tris $\mathrm{HCl} \mathrm{pH}$ 7.5, were mixed at equimolar concentrations of $10 \mu \mathrm{M}$ in a PCR tube. The oligonucleotides were first denatured by heating at $95{ }^{\circ} \mathrm{C}$ for $3 \mathrm{~min}$, before being subjected to an annealing step with gradual cooling from $94{ }^{\circ} \mathrm{C}$ to $25{ }^{\circ} \mathrm{C}$ at $1-{ }^{\circ} \mathrm{C}$ gradients and an 11-s incubation under each temperature. One microliter of the annealed mix was ligated into the vector pX330 (50 ng) pre-digested with BbsI using 5 units of T4 ligase (Cat. EL0011, Thermo Fisher Scientific) in a 10- $\mu \mathrm{L}$ reaction system at room temperature (RT, $20-22^{\circ} \mathrm{C}$ ). The ligation products were transformed into DH5 $\alpha$ and the resulting colonies were sent for DNA sequencing to identify desired constructs. 
above, $0.16 \mu \mathrm{g}$ of pMaxGFP (as a FACS marker later), $16 \mu \mathrm{L}$ of P3000 (Cat.

L3000008, Thermo Fisher Scientific), and $250 \mu \mathrm{L}$ of Opti-MEM medium (Cat. 31985070, Thermo Fisher Scientific) were mixed and added to a separately prepared mix containing $16 \mu \mathrm{L}$ of Lipofectamine 3000 and $250 \mu \mathrm{L}$ of Opti-MEM medium. After incubation at RT for $15 \mathrm{~min}$, the mixture was added dropwise to a 6-cm dish containing HEK293 cells with 70\%-90\% confluency. Cells were cultured in a 5\% $\mathrm{CO}_{2}$ incubator at $37^{\circ} \mathrm{C}$ in a Dulbecco's Modified Eagle Medium (DMEM) supplemented with $10 \%$ fetal bovine serum (FBS). After $24 \mathrm{~h}$, cells were washed with $2 \mathrm{~mL}$ of PBS and digested with $0.5 \mathrm{~mL}$ of $0.1 \%$ trypsin (Cat. 25200056, Thermo Fisher Scientific) for $3 \mathrm{~min}$ at $37^{\circ} \mathrm{C}$ before being re-suspended in $3 \mathrm{~mL}$ of DMEM and $10 \%$ FBS to saturate trypsin. Cells were then harvested by centrifugation at RT at $300 \mathrm{~g}$ for $5 \mathrm{~min}$, washed with $10 \mathrm{~mL}$ of PBS, and resuspended in $0.5 \mathrm{~mL}$ PBS for fluorescence assisted cell sorting (FACS). A total of $\sim 800,000$ cells were sorted in a BD FACSAria Fusion machine by green fluorescence protein (GFP) and the top 5\% $(\sim 40,000)$ were collected. These cells were serially diluted using DMEM supplemented with $10 \%$ FBS and seeded into 96-well plates such that an average of 2 or 4 cells were contained in each well with $100 \mu \mathrm{L}$ of medium. Single cells were allowed to populate for 10-12 $\mathrm{d}$ in a stationary $\mathrm{CO}_{2}$ incubator. Wells containing a single colony were selected under a microscope, and the selected cells were washed with $30 \mu \mathrm{L}$ PBS before being treated with $30 \mu \mathrm{L}$ of $0.1 \%$ trypsin for $2 \min$ at $37^{\circ} \mathrm{C}$. After this, cells were resuspended in $200 \mu \mathrm{L}$ of DMEM and 10\% FBS and divided into two parts $(160 \mu \mathrm{L}$ and $70 \mu \mathrm{L})$ for further culturing and PCR-identification, respectively. The alternation of genome was 
screened using the following primer pairs: GPAA1, 5'- AGGACTCCGGGTTTAGGT CT -3' and 5'- GTAGCCCAATCAAGGACCCC -3'; PIGK, 5'- TAAGCGATCTGC CCTACCAC -3' and 5'- CCCACAGGGAAGAATTC GGG -3'; PIGS, 5'- GGCGAA ATGGGTGTCATGTG-3' and 5'- GGCATGCAGATT TCCCTCCT-3'; PIGT, 5'GACTGTGCTTAAGGAGGGCA -3' and 5'- AGCCTAA CGTTGCCAAACCC -3'; PIGU, 5'- GCACAAAATGGTCCGGCAG-3' and 5'- AGGCCCATTAAGGCCAAG TT-3'. Cells pellets were resuspended into a mix containing $10 \mu \mathrm{L}$ of $0.1 \mathrm{mM}$ EDTA, $1 \%$ Tween-20, $1 \times$ Taq polymerase buffer $(50 \mathrm{mM} \mathrm{KCl}, 2 \mathrm{mM} \mathrm{MgCl} 2,20 \mathrm{mM}$ Tris $\mathrm{HCl} \mathrm{pH} \mathrm{8.4),} \mathrm{and} 1 \mathrm{mg} \mathrm{mL}^{-1}$ protease $\mathrm{K}$. The mixture was heated successively at 56 ${ }^{\circ} \mathrm{C}$ for $2 \mathrm{~h}$ and $95^{\circ} \mathrm{C}$ for $30 \mathrm{~min}$ in a thermocycler, before PCR amplification for 40 cycles. Compared to the wild-type cells, PCR products from knockout (KO) cells lacked a large band (GPAA1, 2 kb; PIGS, 2.7 kb; PIGK, 3.7 kb; PIGT, 3.4 kb; PIGU, $2.3 \mathrm{~kb}$ ) but showed a smaller band (GPAA1, $1 \mathrm{~kb}$; PIGS, $0.7 \mathrm{~kb}$; PIGK/PIGT/PIGU, $1.2 \mathrm{~kb}$ ) owing to the deletion. The genomic deletions were further verified by DNA sequencing of the PCR products using the abovementioned primers.

To confirm the lack of GPI-AP surface expression in the individual KO cells, cells identified as positive above were allowed to grow for two more passages before being analyzed by flow cytometry (see the section below). Cells that 1) lacked CD59 on cell surface, and 2) the surface expression of CD59 could be restored by ectopic expression of the corresponding subunits were either used for further flow cytometry assays or cryo-preserved in 10\% DMSO, 45\% FBS in DMEM for long storage. 
performed in tandem. Defective GPI-T in this cell line was confirmed by DNA sequencing as above, and by the lack of CD59 staining upon transfection of combination of any four, but not all five, subunits.

\section{Flow cytometry}

Wild-type or GPI-T KO HEK293 cells were cultured in Dulbecco's Modified Eagle Medium (DMEM) supplemented with $10 \% \mathrm{FBS}$ at $37{ }^{\circ} \mathrm{C}$ in 24 -well plates inside a $\mathrm{CO}_{2}$ stationary incubator. Plasmids $(0.5 \mu \mathrm{g}$ of single plasmids, or a total of $0.5 \mu \mathrm{g}$ of multiple plasmids) were mixed with $1 \mu \mathrm{L}$ of $\mathrm{P} 3000,25 \mu \mathrm{L}$ of Opti-MEM medium, and added to a separate mix containing $1.5 \mu \mathrm{L}$ Lipofectamine 3000 and $25 \mu \mathrm{L}$ Opti-MEM medium. Transfection was carried out as outlined above. Two days after transfection, cells were washed with PBS, treated with trypsin as in the previous section, and washed and resuspended in 0.5 mL PBS. Phycoerythrin (PE)-labeled CD59 antibody (12-059642, Thermo Fisher Scientific, $1: 500$ dilution) was incubated with the cells for $15 \mathrm{~min}$ in dark. Cells were washed with PBS and resuspended in $\sim 0.3 \mathrm{~mL}$ of PBS for flow cytometry (Beckman CytoFlex LX) monitored at two wavelength pairs (488/525 for GFP, 561/585 for PE). Cells (typically 40,000) were gated using the GFP channel (from expression of TGP-tagged GPI-T subunit(s)) and analyzed for positive signal for the PE channel (for surface staining of CD59) using the software FlowJo (BD Life Sciences).

For the apparent activity of GPI-T mutants, the percentage of the immune staining 
of CD59 in singly KO-cells transfected with the mutant was normalized to the negative control (the same cell line transfected with the TGP-tagged Patched, an unrelated membrane protein) and the positive control (the same cell line transfected with plasmids carrying the wild-type subunit gene). The expression and integrity of all mutants (TGPtagged) was also separately confirmed by SDS-PAGE in-gel fluorescence ${ }^{37}$. Data reported in this work were from three independent experiments (Supplementary Data 1).

\section{GPI-T expression and purification}

GPI-T was expressed in Expi293 cells co-transfected with five plasmids carrying all subunits using polyethylenimine (PEI). Briefly, $0.5 \mathrm{~L}$ of cells (>95\% viability) at a density of $2 \times 10^{6} \mathrm{~mL}^{-1}$ were diluted 2 times and cultured at $37^{\circ} \mathrm{C}$ in a $3 \mathrm{~L}$ flask in a $\mathrm{CO}_{2}$ incubator to re-reach $2 \times 10^{6} \mathrm{~mL}^{-1}$ (typically takes one day). Six milligrams of plasmids and $12 \mathrm{mg}$ of PEI were mixed in $100 \mathrm{~mL}$ of medium for $20 \mathrm{~min}$ at $\mathrm{RT}$ before being added into $1 \mathrm{~L}$ of cell culture. Sodium valproate (Cat. P4543, Sigma) was added to a final concentration of $2 \mathrm{mM}$. Cells were harvested after $48 \mathrm{~h}$ by centrifugation at 1,500 $\mathrm{g}$ for 15 min, washed once with PBS buffer, flash-frozen in liquid nitrogen, and stored at $-80{ }^{\circ} \mathrm{C}$ until use.

GPI-T was purified by tandem affinity chromatography followed by gel filtration.

All steps were performed at $4{ }^{\circ} \mathrm{C}$. Cells from $4 \mathrm{~L}$ of culture were re-suspended with solubilization buffer containing $1 \% \mathrm{LMNG} / 0.1 \% \mathrm{CHS}, 150 \mathrm{mM} \mathrm{NaCl}, 1 \mathrm{mM}$ PMSF, $1 \times$ protein inhibitor cocktail (Cat. B14001, Bimake), and $50 \mathrm{mM}$ Tris-HCl pH 8.0 and 
stirred gently for $2 \mathrm{~h}$. Cell debris were removed by centrifuging at $4,4200 \mathrm{~g}$ for $1 \mathrm{~h}$. The supernatant was collected, mixed with $4.5 \mathrm{~mL}$ pre-equilibrated Strep Tactin beads (Cat. SA053100, Smart-lifesciences) and agitated gently for $2 \mathrm{~h}$. The beads were packed into a gravity column (Cat. 7321010, Bio-Rad) and washed with 5 column volume (CV) of $0.01 \%$ LMNG, $0.001 \%$ CHS and $0.1 \%$ Digitonin before being incubated with $0.2 \%$ digitonin in Buffer $\mathrm{A}(150 \mathrm{mM} \mathrm{NaCl}, 20 \mathrm{mM}$ Tris $\mathrm{HCl} \mathrm{pH} \mathrm{8.0)} \mathrm{for} 1 \mathrm{~h}$. The beads were then washed with $1.5 \mathrm{CV}$ of $0.2 \%$ digitonin and $5 \mathrm{CV}$ of $0.1 \%$ digitonin in Buffer A before being eluted with $5 \mathrm{mM}$ d-desthiobiotin and $0.1 \%$ digitonin in Buffer A. The pooled fractions were adjusted to contain $10 \mathrm{mM}$ imidazole before incubated with $1 \mathrm{~mL}$ of Ni-NTA beads (Cat. 1018401, Qiagen) for $1 \mathrm{~h}$ with mild agitation. The beads were packed into a gravity column, washed with $5 \mathrm{CV}$ of $10 \mathrm{mM}$ imidazole, before being eluted using $0.3 \mathrm{M}$ imidazole in $0.1 \%$ digitonin in Buffer A. The Pooled fractions were concentrated with a 100-kDa cut-off concentrator (Cat. UFC810096, Merck millipore) and further purified with gel-filtration on a Superose 6 10/300 GL column (Cat. 290915-96, Cytiva) in a running buffer containing $0.1 \%$ digitonin in Buffer A. Peak fractions were pooled and concentrated to $14-20 \mathrm{mg} \mathrm{mL}^{-1}$ for cryo-EM grid preparation. Protein concentration was determined by measuring $\mathrm{A}_{280}$ using a theoretical molar extinction coefficient of 596,940 $\mathrm{M}^{-1} \mathrm{~cm}^{-1}$ assuming an equimolar stoichiometry.

\section{Cryo-EM sample preparation and data collection}

Purified GPI-T complex (2.5 $\mu \mathrm{L})$ was applied onto glow-discharged Quantifoil Au R1.2/1.3 (300 mesh) grids, and blotted with filter paper for $3 \mathrm{~s}$ with a blotting force of 
5 at $4{ }^{\circ} \mathrm{C}$, with $100 \%$ humidity in a Vitrobot Mark IV (FEI) chamber before being cryocooled in liquid ethane.

Grids were loaded in a Titan Krios cryo-electron microscope (Thermo Fisher) operated at $300 \mathrm{kV}$ with a $50-\mu \mathrm{m}$ condenser lens aperture, spot size 5 , magnification at $165,000 \times$ (corresponding to a calibrated sampling of $0.85 \AA$ per physical pixel), and a K2 direct electron device equipped with a BioQuantum energy filter operated at $20 \mathrm{eV}$ (Gatan). Movie stacks were collected automatically using EPU2 software (Thermo Fisher) with the $\mathrm{K} 2$ detector operating in counting mode at a recording rate of 5 raw frames per second and a total exposure time of $5 \mathrm{~s}$, yielding 25 frames per stack and a total dose of $52.5 \mathrm{e}^{-} / \AA^{2}$.

\section{Cryo-EM data processing}

Cryo-EM data were processed using Relion (v3.1) ${ }^{54}$ and CryoSPARC (v3.1) ${ }^{55}$. The frame motion of a total of 4,705 images stacks was corrected by MotionCor2 ${ }^{56}$ wrapper in Relion. Exposure-weighted averages were then imported to CryoSPARC and the contrast transfer function parameters for each micrograph were estimated by CTFFIND4 ${ }^{57}$. 2D classification template was generated from a small set of particles via blob-picking. A total of 2,959,791 particles were autopicked using this template and extracted with a box size of 280 pixels, and subjected to several rounds of $2 \mathrm{D}$ classification and heterogeneous refinement (3D classification) to remove contaminants or poor-quality particles. A set of 329,617 good GPI-T particles was obtained and 
converted for Bayesian polishing in Relion, which was subsequently imported back to CryoSPARC for heterogeneous refinement, enabling the production of a $2.66 \AA$ map from 179,871 particles by non-uniform refinement (NU-refine). A further round of heterogeneous refinement (3D classification) with phase randomized reference maps (20/30/40 $\AA$ ) narrowed the dataset down to 151,590 particles, which was used to generate the final $2.53 \AA$ map via local refinement with a membrane micelle-removed mask. Resolution of these maps was estimated internally in CryoSPARC by goldstandard Fourier shell correlation using the 0.143 criterion. Details for data processing are in Supplementary information (Fig. S3) and Table S1.

\section{Model building}

The model was ab initio built using $\operatorname{Coot}^{58}$ based on the amino-acid sequence and guided by the cryo-EM density using aromatic residues, glycosylation sites, and disulfide bonds as reference markers. The model was refined with Phenix. real_space_refine ${ }^{59}$, yielding an averaged model-map correlation coefficient (CCmask) of 0.84. Structures were visualized using UCSF ChimeraX1.1 ${ }^{60}$ and PyMol (https://pymol.org/2/).

\section{Data Availability}

The coordinates for GPI-T model have been deposited in the PDB under accession code 7WLD. The cryo-EM density map has been deposited in the Electron Microscopy Data Bank with accession code EMD-32582. Data for Figs. 2d/3d/S7d/S8b are included in 
654 Supplementary Data 1.

655 Supplementary Information

656

657

Fig. S1-S9

658

659 Table S1 

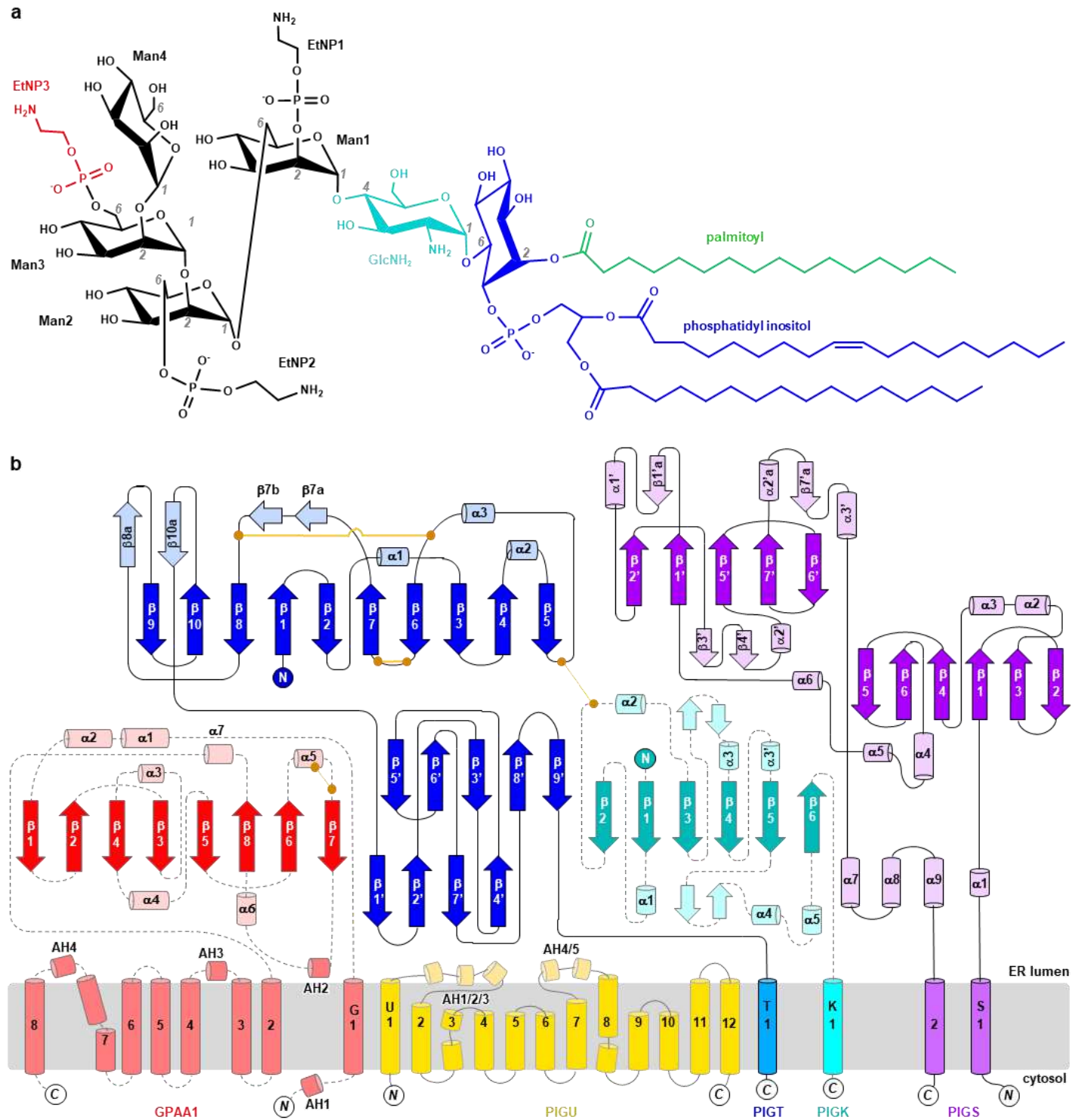

Fig. S1. Chemical structure of GPI and topology of GPI-T. a The GPI core contains a phosphatidyl inositol (blue), a palmitoyl chain (green), a glucosylamine $\left(\mathrm{GlnNH}_{2}\right)$ (cyan), and four mannoses (Man) with possible modification of ethanolamine phosphate (EtnP) on Man1/2. The reactive EtnP3 on Man3 is colored red. The numbering of the carbon atoms on the sugar ring is indicated by a grey number when appropriate. b Topology of GPI-T drawn with structural knowledge from this study. 

are numbered to match the description in the main text and Fig. S2. AH, amphipathic 671 helix. N/C indicates the $\mathrm{N}$ - and $\mathrm{C}$-terminal of subunits. 
a

H. sapiens (human, PIGK)

s. cerevisiae(yeast, Gpi8p)

H. sapiens (Q92643)

s. cerevisiae (P49018)

H. sapiens

s. cerevisiae $(45.08 / 63.78)$

H. sapiens

S. cerevisiae

H. sapiens

S. cerevisiae

\section{$\longrightarrow \beta 1 \longrightarrow \alpha .1-\beta 2$}

28 SHIEDQAEQF FRSGHTNNWA VLVCTSRFWE NYRHVANTLS VYRSVRRLGI PDSHIVIMLA DDMACNPRNP KPATVFSHKN MELNVYGDDV 3 --NNTDAAHE VIATNTNNWA VLVSTSRFWE NYRHMANVLS MYRTVKRIGI PDSQIILMLS DDVACNSRNL FPGSVFNNKD HAIDLYGDSV

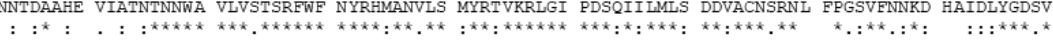

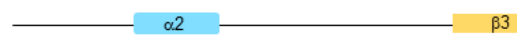

\section{3}

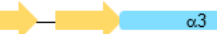

$\beta 4$

118 EVDYRSYEVT VENFLRVLTG RIPPSTPRSR RLLSDDRSNI LIYMTGHGGN GFLRFQDSEE ITNIELADAF EQMWOKRRYN ELLFIIDTCO 11 EVDYRGYEVT VENFIRLLTD RWTEDHPKSK RLLTDENSNI FIYMTGHGGD DFLKFQDAEE IASEDIADAF QQMYERKRYN EIFFMIDTCQ

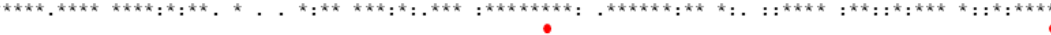

$\alpha 3^{\prime}-\beta 5$

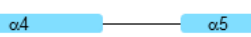

$\beta 6$

RYTFYVLEE FEINPASOTN MNDLFOVCPR SLCVSTPGHR TDLFORDPKI 201 ANTMYSRFYS PNILAVGSSE MDESSYSHHS DVEIGVAVID RFTYYCLDFL EQIDKNSTLT LQDLFDSFTF ERIHSHVGVR TDLFDRNPSE

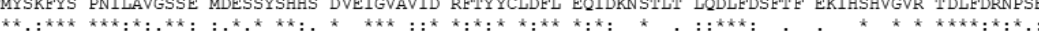
298 VLITDFFGSV R-------- ---------K VEITTETIKL QQ-DSEIMES SYKEDQMDEK LMEPLKYAEQ LPVAQIIHQR PRLKDWHPPG 291 VLITDFFANV QNVIPDDSKP LSVSHYHHYK DHIDTAQYEL NNNVLDLALE TYRKNNQSSR IEKRIKDIKS TSVLDVDIDS NECFFTSFRQ $:^{\star}:: \quad:: \quad:^{\star}:::: .^{*}:::^{*} \quad: . .^{*}:: \quad:$

\section{TMHK1}

369 GFILGLWALI IMVFFRTYGI RHMRFIF---

381 SATIILALIV TILWFMLRGN TARATYDLYT

SATIILALIV TILWFMLRGN T
$: *:::::^{*} *$

H. sapiens (human, PIGT)

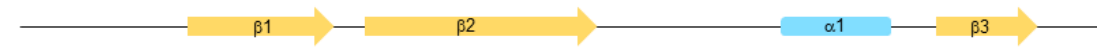
22 --------LA EPPRDSLREE LVITPLPSGD VAATFQFRTR ------WDSE LQREGVSHYR LFPRALGQLI SKYSLRELHL SFTQGFWRTR

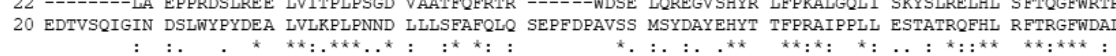

\section{$\beta 4$} $\alpha 2$ $\beta 5$ 98 YWGPPFLQAP SGAELWVWFQ DTVTDVDRS- ----WKELSN VLSGIFCASI NFIDSTNTVT PTASFRPLG- --LANDTDHY FLRYAVLPRE 110 SWGQLPHAGK EAGASGVELW SQVQAMDQEO AFHNWKLLSN SLSGLFCSSL NFIDESRTTF PRRSYASDIG APLFNSTERL YLMRASLPNE

H. sapiens (Q969N2)
S. cerevisiae( 138875$)$

$$
\alpha 3
$$

$$
\beta 6
$$$$
\beta 7
$$
$*^{*} \cdot:::^{*}{ }^{* * *} \cdot{ }^{*}$

\section{H. sapiens}

180 VVCTENLTPW KKLLPCSSKA GLSVLIRADR LFHTSYHSOA VHIRPVCRNA RCTSISWELR OTLSVVFDAF ITGQGKKD--

PICTENLTPF IRLLPTRGKS GLTSLLDGHK LFDSLWNSIS LDIATICSED EDALCHYEMD ARIEMVTHVP SALARGERPI PKPLDGNTLR

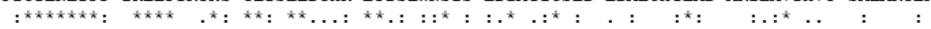

$-\beta-\beta 7 \mathrm{~b}-\beta 8-\beta 8 \mathrm{a}-\beta 9-\beta 10-\beta 10 \mathrm{a}$

H. sapiens

s. cerevisiae

H. sapiens

s. cerevisiae

H. sapiens

s. cerevisiae

H. sapiens

s. cerevisiae 258 WSLFRMFSRT LTEPCPLASE SRVYVITTY NQDNETLEVH PPPTTTYQDV ILGTRKTYAI YDLLDTAMIN NSRNLN---- ------IQI
290 CDTDKPFDSY QCFPLPEPSQ THFKLSQLFA RPINNGNLFA NRPTRICAEV DRSTWTAFLS VDDTIFSTHD NCFDLSNDQN EGGSGDFIL

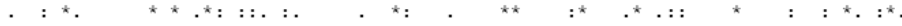

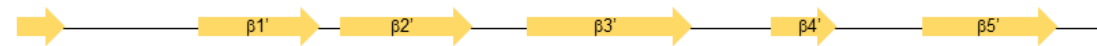

337 KWKRPPENEA PPVPFLHAQR YVSGYGLQRG ELSTLLYNTH PYRAFPVLLL DTVPWYLRLY VHTLTITSKG KENKPS---- -YIHYQPAOD 380 ESTDTTKVTP IVPVPIHVSR SLTGNGQDRG GMRIVFHNDN -DTPVRLIYF ESLPWFMRVY LSSLQITSTT SPQLQENDII LDKYYLQAAD

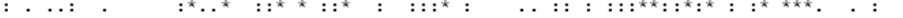

$-\beta 6^{\prime}-\beta 7^{\prime}-\beta 8^{\prime} \longrightarrow \beta 9^{\prime}$ 422 RLQPHLLEML IQLPANSVTK VSIQFERALL KWTEYTPDPN HGFYVSPSVL SALVPSMVAA KPVDWEESPL FNSLFPVSDG SNYFVRLYT 469 RKRPGHLEFT MLIPANTDIV MTYQFDRALL QFAEYPPDAN HGEEIDAAVI TVLS------ ---------- -------LES SSSLYEMRTS

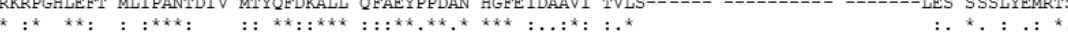

\section{TMHT1}

512 PLLVNLPTPD FSMPYNVICL TCTVVAVCYG SFYNLLTRTE HIEEPR---- -TGGLAKRLA NLIRRARGVP PL--536 TLLLSLSTPD FSMPYNIIL TSTIMGLIFG MLYNLMVRM VTVEEADRIT LQSGLKYRLL RLKERFLGRR RTRTD 
c

H. sapiens (huamn, PIGU)

s. cerevisiae(yeast, Gablp)

H. sapiens $(09 \mathrm{H} 490)$

s. cerevisiae (P41733)

H. sapiens

S. cerevisiae $(28.58 / 49.38)$

H. sapiens
S. cerevisia

H. sapiens

s. cerevisiae

d

H. sapiens (human, PIGS)

S. cerevisiae(yeast, Gpil7p)

H. sapiens (Q96S52)
S. cerevisiae(004080) 91 EIPLKYKMRI RCRFQRAYRR ALDHEEEALS SGSVQEAEAM LDEPQEQAEG SLTVYVISEH SSLLPQDMMS YIGPKRTAVV RGIMHREAFN

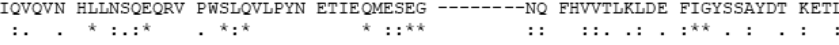

$\alpha 4$ - $\alpha .5-\beta 1^{\prime} \mathrm{a}$

H. sapiens

S. cerevisiae $(22.18 / 38.78)$ 181 IIGRRIVQVA QAMSLTEDVI AAALADHLPE DKWSAEKRRP LKSSLGYEIT FSLLNPDPKS HDVYWDIEGA VRRYVQPFLN ALGAAGNFSV 43 AVLSNDLPFF VAQTLVEHTF QLEWT-HLNK TCEGVSTNND VAISYDPNIH LSVTLLSGDG NPVAWEIEPT LTDYFSPFRK FLSPLVNETV $\beta 2^{\prime} \longrightarrow \beta 3^{\prime}-\beta 4^{\prime} \longrightarrow \beta 5^{\prime} \longrightarrow \beta 6^{\prime}$

H. sapiens

s. cerevisiae

271 DSQILYYAML GVNPREDSAS SSYYLDMHSI PHVINPVESR LGSSAASLYP VLNFLLYVPE LAHSPLYIQD KDG------- APVATNAFHS

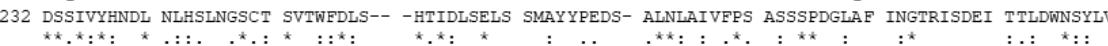

$-\beta 7^{\prime}-\alpha 2^{\prime} \mathrm{a}-\beta 7^{\prime} \mathrm{a}-\alpha 3^{\prime}-\alpha 7$

H. sapiens
S. cerevisiae

354 PRWGGIMVYN VDSKTYNASV LPVRVEVDMV RVMEVFLAQL RLLFGIAQPQ LPPRCLLSGP TSEGLMTWEL DRLLWARSVE NLATATTTLT FEDEPMMY RFATDIFQLL GLT

$\alpha 7-\alpha .8$ - $\alpha 92$

H. sapiens
S. cerevisia

444 SLAQLLGKIS NIVIKDDVAS EVYKAVAAVQ KSAEELASG- --HLASAFVA SQEAVTSSEL AFFDPSLLHL LYFPDDQRFA IYIPLFLPMA 396 KLTQQFQGMS IPREVSDNVI EALDLRLQII DLLNDPGKGG DIVWNNALHL SNELVRLCER AFFNGEMVQQ NFFPQEHMIA VYLPLLGPIS (

H. sapiens
5. cerevisia

(2010

531 VPILLSLVRI FLETRRSWRK --------PE RTD------- --------

486 AVMFFGFYNV MRERNORSKK NGTEREVARE RLELKEAORL HAIDGEDEL 

MALIERLHRR IVDMGLVPRI IALLPVISML CALFGFISIA ILPMDGQYRR TYISENALMP SQAYSYFR-- ESEWNILRGY RSQIKEMVMM

$-\alpha 2-\beta 1-\beta 2-\beta 3-\alpha 3$ - $-\beta 3$ 88 PVAWLERTMR SVGLEVYTQS FSRRLPFPDE THERYMVSGT NVYGILRAPR AASTESLVLT VPCGS--DST NSQAVGLLLA LAAHFRGQIY 89 TSMERNNLMG SWLQEFGTKT AIYENEQ--- ------YGE TLYGVMHAPR GDGTEAMVLA VPWFNSDDEF NIGGAALGVS LARFFSRWPV

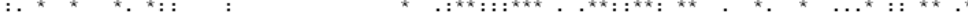

- $\beta 4$ - $\alpha 4$ - $\beta 5$

6 WARDIVFLVT EHDLIGTEAW LEAYHDVNVT GMQSSPLQGR AGAIQAAVAL ELSSDVVTSI DVAVEGLNGQ LPNLDLLNLF QTFCQKGGLI

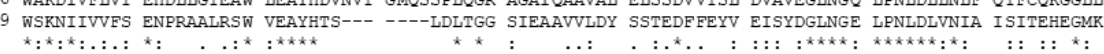

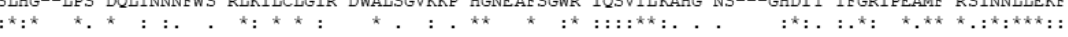

H. sapiens
S. cerevisiae 354 HOSFFLYLLP GLSRFVSIGL YMPAVGELLL VLGLKALELW MQLHEAGMGL EEPGGAPGPS VPLPPSQGVG LASLVAPLLI SQAMGLALYV

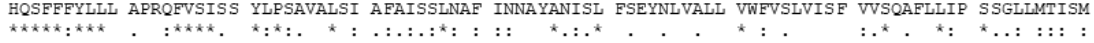

H. sapiens - AHG3 TMHG4

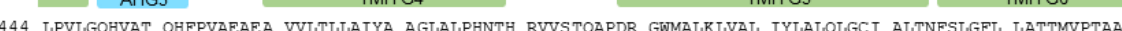
427 ASCFLPLILS RKIHISEPLS YRLKNVAFLY FSLVSTS-LL MINFAMALI GTLAFPMTFV KTIVESSSEH EVTTQSSNPI RTEPRDEIEL

S. cerevisiae

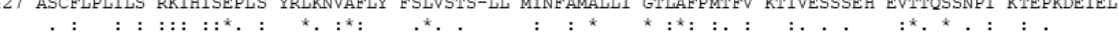

H. sapiens
S. cerevisia

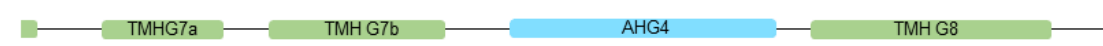

534 LARPHGPRTL YAALLVLTSP AATLLGSLFL WRELQEAPLS LAEGWQLFLA ALAQGVLEHH TYGALLFPLI SLGLYPCWLL FWNVLFWK-516 VENHMDTTPA TPQQQRQRL NLVLLILTNP FISITLFGLF FDDEFHGDI IN-KLVSAWL DLKCWSWFV CIGWLPCWLI ILASSFESKS

H. sapiens
S. cerevisia

605 VVVRSKERQS
682

Fig. S2. Sequence alignment of GPI-T from human (Homo sapiens) and yeast (Saccharomyces cerevisiae). a PIGK. The catalytic dyad residues are marked with red dots. b PIGT; c PIGU; d PIGS; e GPAA1. Uniprot IDs are included along with the sequence identity/similarity compared to the human orthologues. Secondary structure elements of the human GPI-T are labeled based on the 3D structure determined in this work. Asterisk, colon, and dot indicate identical, conserved, and semi-conserved substitutions, respectively. Secondary structure segments are number sequentially unless they are called differently in the text for ease of discussions in some cases such as structure comparison and for sub-domain descriptions. 

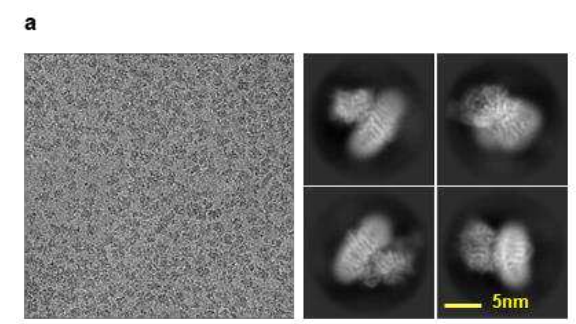

b
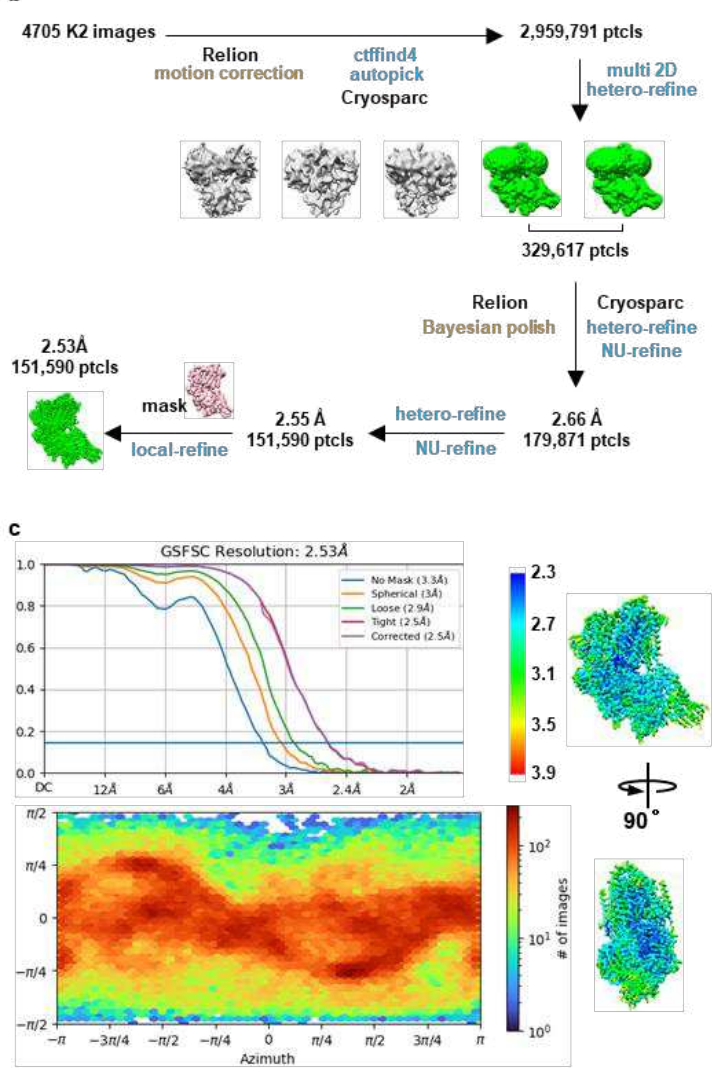

$\rightarrow$
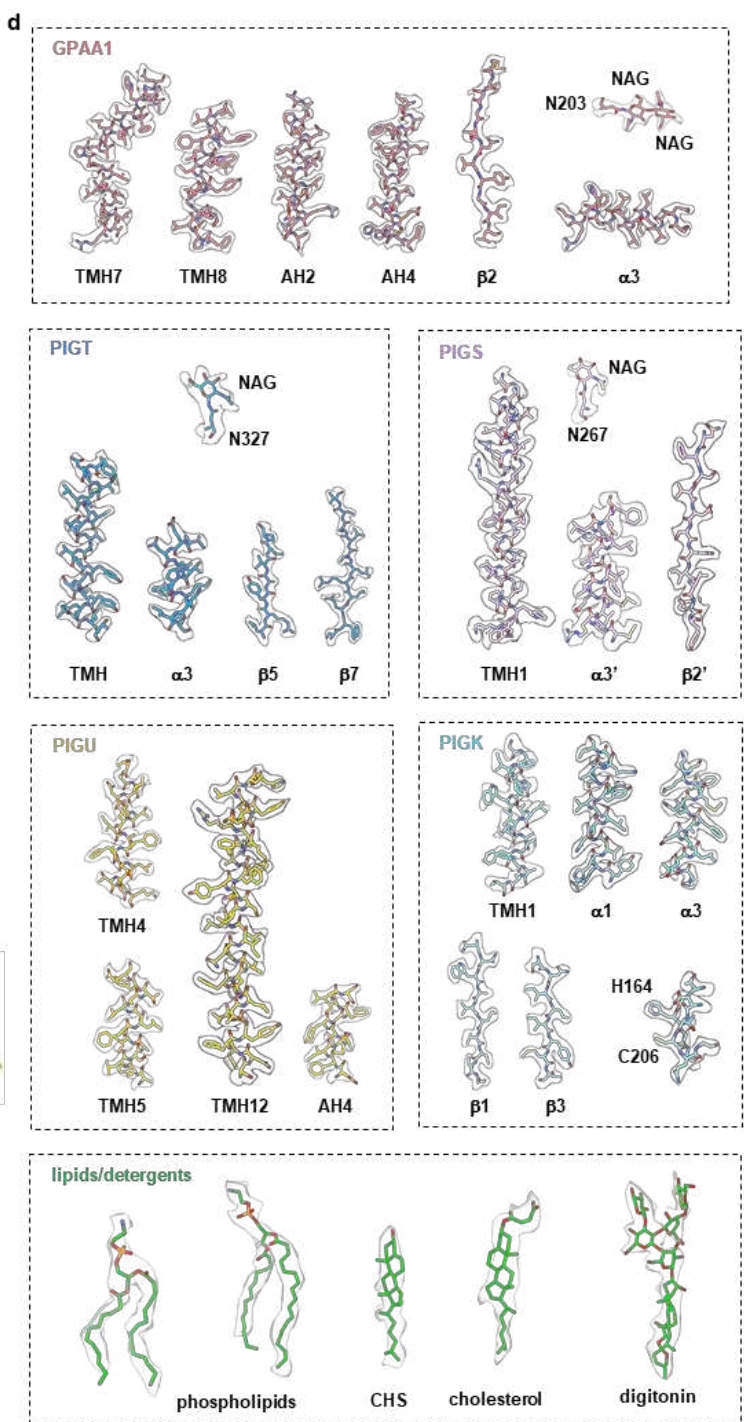

Fig. S3. Cryo-EM data processing and high-quality density/model fitting exemplary views. a Representative cryo-EM micrograph of GPI-T and selected 2D class averages. b The workflow of classification and refinement. c The nominal resolution of GPI-T was determined by the 'gold-standard' FSC curve using the $\mathrm{FSC}=0.143$ criterion, and angular distribution heatmap at the bottom panel calculated in Cryosparc. Local resolution evaluation was shown on right. d Cryo-EM map density and model of representative protein parts, glycosylation sites, and lipids and detergents. 

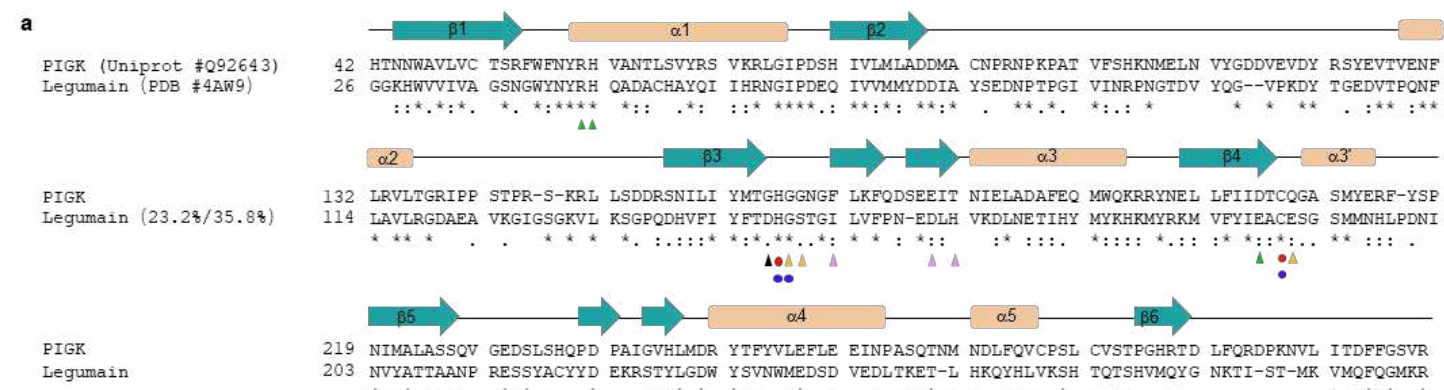
10.
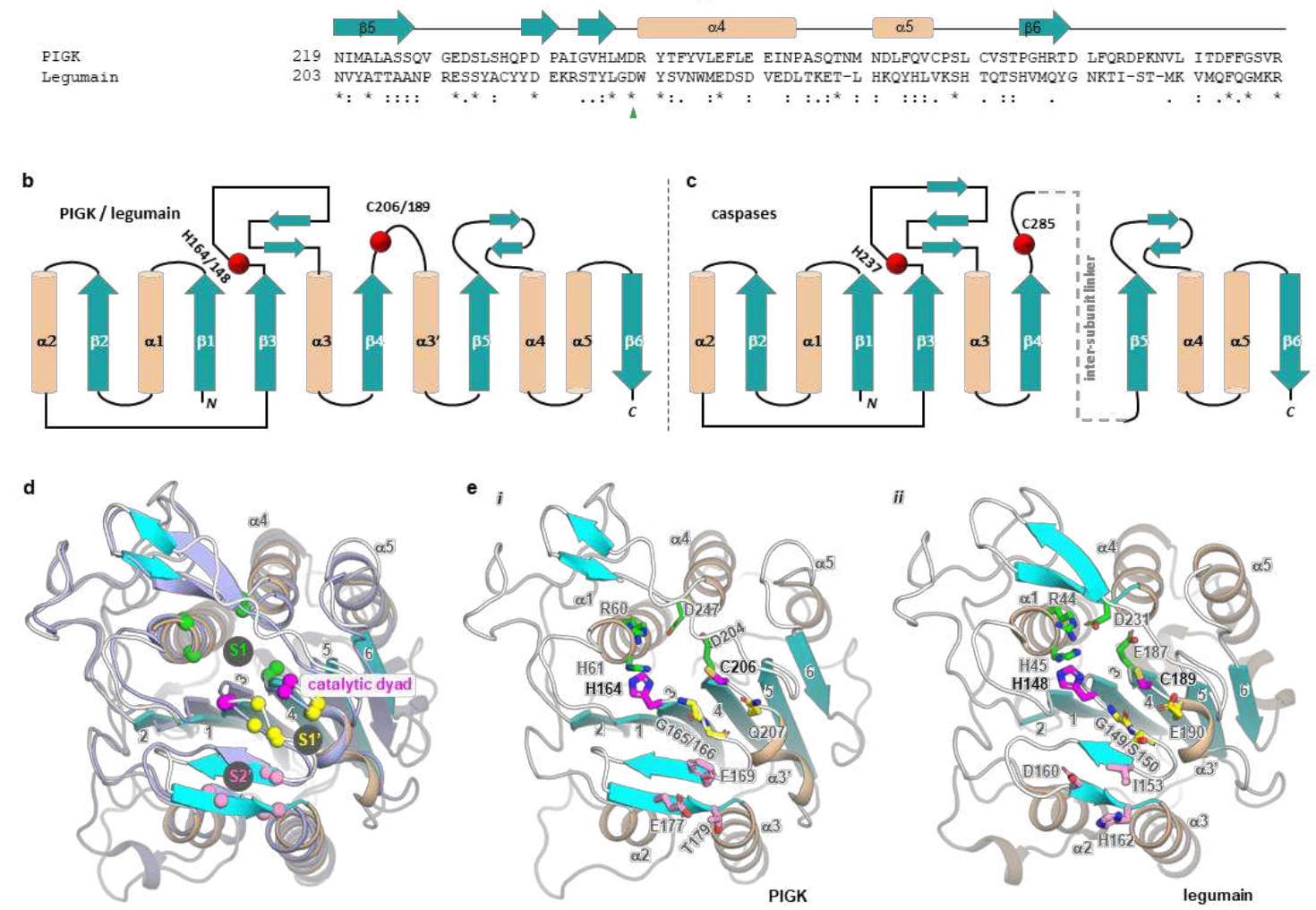

Fig. S4. PIGK shares sequence and structure similarity with legumains and

caspases. a Sequence alignment between PIGK and legumain. Asterisk, colon, and dot indicate identical, conserved, and semi-conserved substitutions, respectively. Red

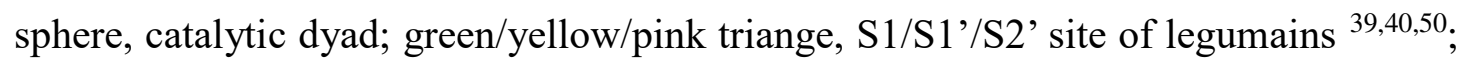
blue dots, oxyanion hole-residues in legumains; black triangle, the aspartate succinimide in legumains ${ }^{50} . \mathbf{b}, \mathbf{c}$ PIGK shares a similar topology with legumains (b) ${ }^{40}$ and caspases $(\mathbf{c})^{38}$. The linker region that was removed in the mature caspases is shown as a dashed line. d, e High structural similarity (Z-score of 29.6 and C $\alpha$-RMSD of 2.3

$\AA$ by the DALI server) ${ }^{41}$ between PIGK and legumains as revealed by superposition 
comparison (e). The six $\beta$-strands (1-6) in the central sheet and connecting a-helices $(\alpha 1-\alpha 6)$ are labeled. The catalytic dyad and $\mathrm{S} 1 / \mathrm{S} 1^{\prime} / \mathrm{S} 2^{\prime}$ residues are shown as $\mathrm{C} \alpha$ spheres (d) or sticks (e) and colored magenta, green/yellow/pink, respectively. 

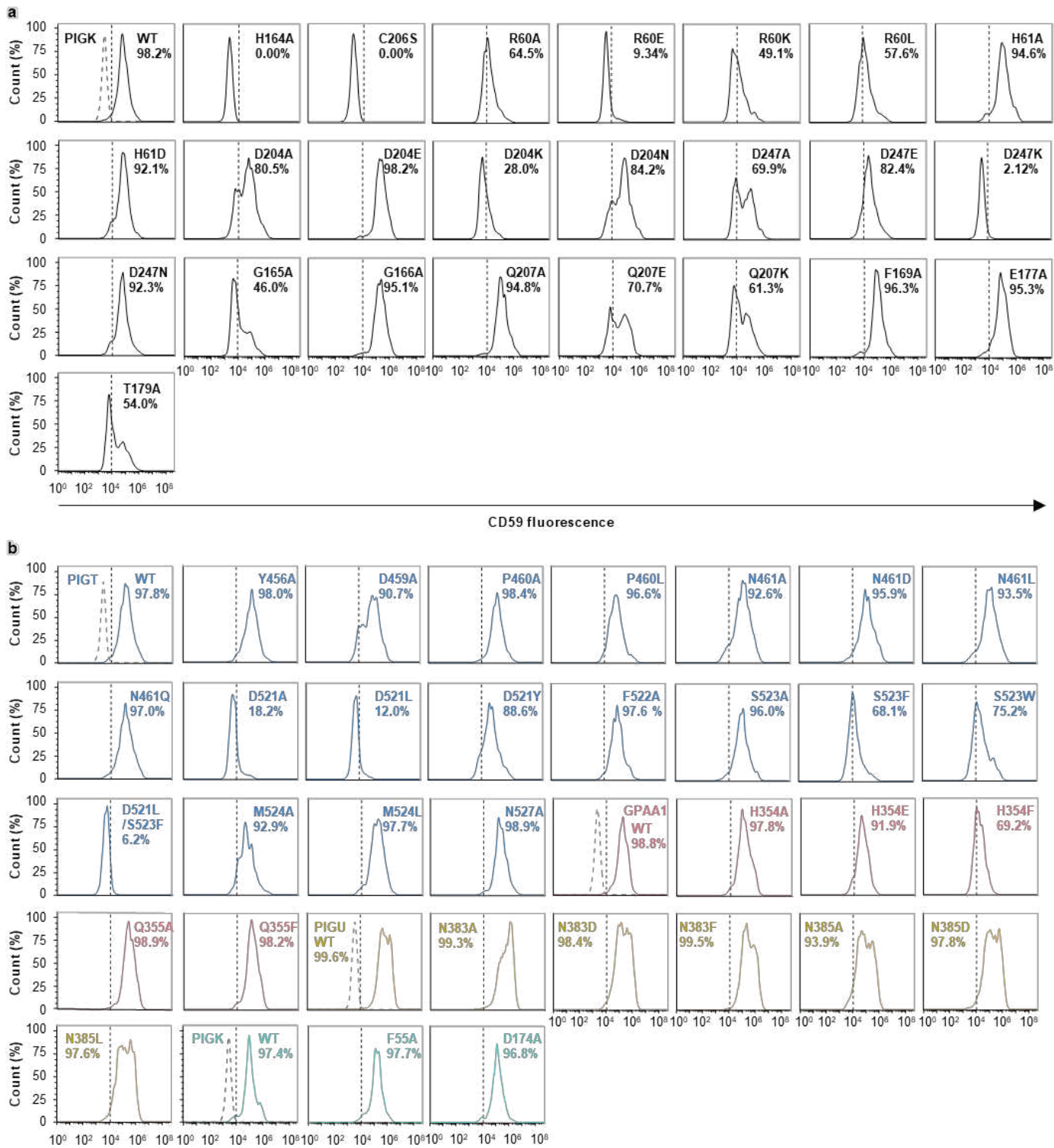

CD59 fluorescence

Fig. S5. Typical fluorescence-activated cell sorting (FACS) results for the constructs described in the main text. a Representative FACS results of three independent experiments for the PIGK mutants (the catalytic dyad and S1/S1'/S2' sites).

b Representative FACS results of three independent experiments for the mutants of the GPI-binding site residues. The percentage shown in this figure represents the subpopulation of CD59-positive cells (as the function of GPI-AP processing) in the 

percentage shown in this figure was normalized using a negative control (grey dashed line and the vertical dash line, cells expressing an unrelated GFP-tagged membrane protein) and a positive control (cells expressing the wild-type). The expression and integrity of the subunits were also separately verified by SDS-PAGE in-gel fluorescence. 

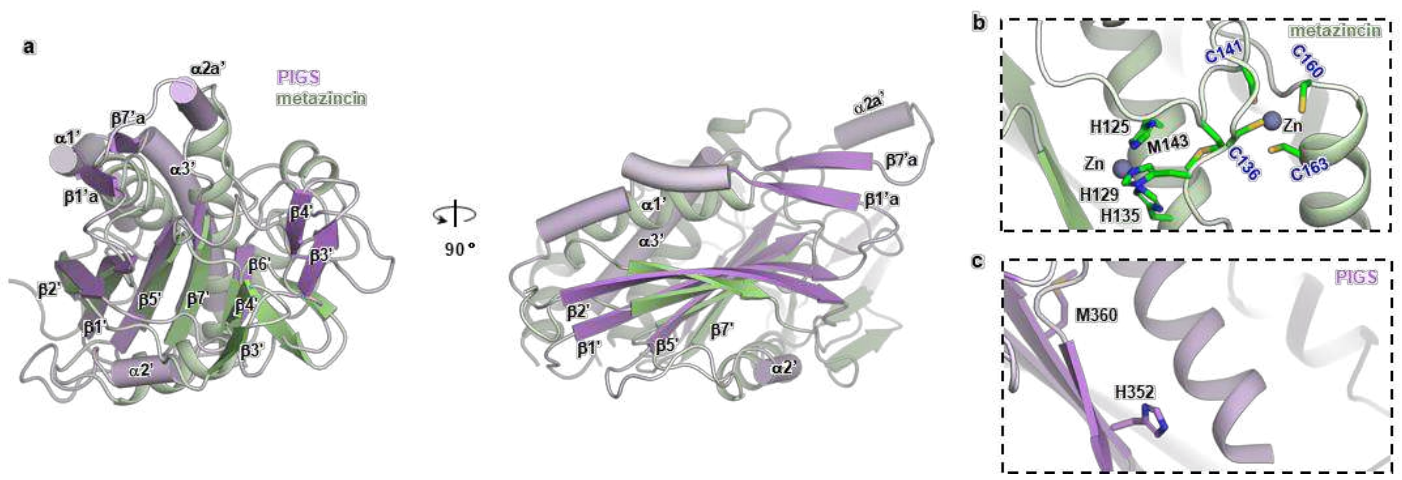

Fig. S6. PIGS contains a protease-like domain. a The PIGS domain containing residues 222-398 (purple, cylinder) shares structural similarity with the metazincin AmzA (green, cartoon, PDB ID 2X7M) with a Z-score of 5.9 and C $\alpha$ RMSD of $3.6 \AA$ ) PIGS (c) as only one histidine and one methionine were found in PIGS in the vicinity 734 (within $10 \AA$ ). 

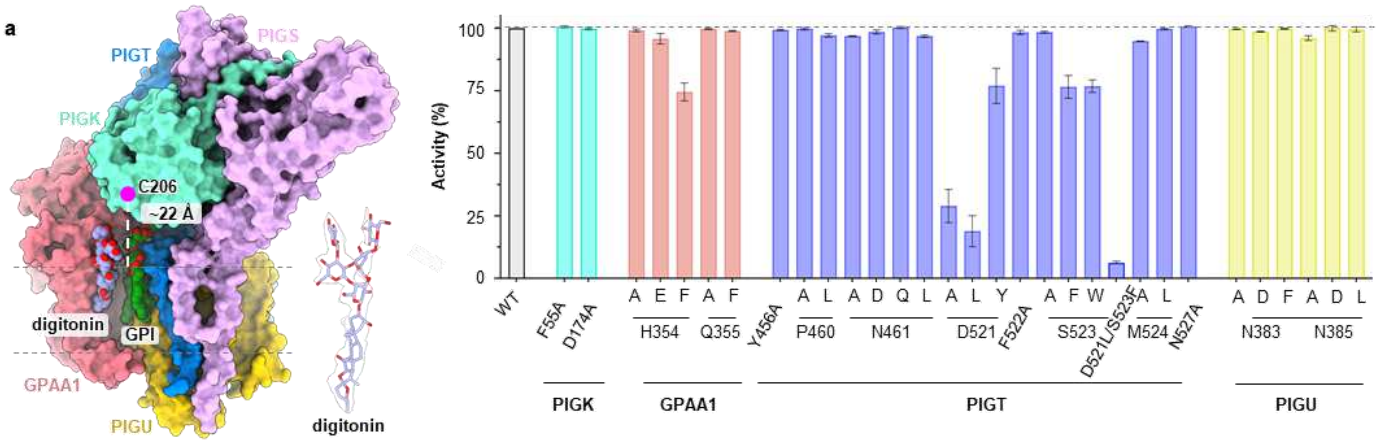

C Homo sapiens human Q92643, PIG Felis catus_cat M3X 6 D5 93.78 Mus musculus mouse Q9CXY9 91.4 Rattus norvegicus_rat_Q5XIP2 91.9 Bos taurus bovin Q3MHZ7 93.98 Equus ferus caballus_horse_F7DVT8_93.4 Vicugna pacos_alpaca_AOA $6 J 3 A X M 5$ 94. 94.58 Rhinolophusferrumequinum_bat_A0A 671 EDE 694.48 Terrapene carolina triunguis_turtle_A0A6 74 JPG6 $86.8 \%$ Xenopus tropicalis_frog_ 005 AQ6 80.7 Danio rerio_ebrafish_otems_Drosophila melanogaster_fruitfly_Q8T4E1_46.8\% Caenorhabditis elegans_round worm_P49048_42.2\% Schizosacoso coses pos -

50 VCTSRFWFNY RHVA

50 VCTSRFWENY RHVA

50 VCTSREWENY RHVA

50 VCTSRFWENY RHVA

50 VCTSREWENY RHVA

5O VCTSRWATY RAVA

5O VCTSRTINY RIVA

SO VCTSREWENY RHVA

50 VCTSRFWENY RHVA

47 VCTSRENTWY RHVA

51 VDARPFWENY RHVA

43 VCTSKTENENY RHVS

43 VSTSREWENY RHMA

31 ISTSRFWENY RHTA

$\beta 1-\alpha 1$

166 GNGFLKFODS EEITNIEL 166 GNGELKFQDS EE ITNIEL 166 GNGFLKFODS EE ITNIEL 166 GNGELKFQDS EEITNIEI 166 GNGFLKFQDS EE ITNIEL 166 GNGFLKFQDS EEITNIEL 166 GNGELKFQDS EEITNIEI 166 GNGFLKFQDS EEITNIEL 166 GNGELKFDDS EEITNVEL 166 GNGFLKFDDS EEITNVEL 163 GNGFLKFQDS EEISMMEL 167 GDGELKFQDS EEITSQEL 150 GDSTRTODS ELINNDL 147 GDGFIKFQDA EELSSEDL

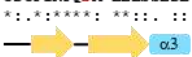

d

Homo sapiens human $096 \mathrm{N2}$, PTG Felis catus_cat_M3WVG3_94.1\% Mus musculus_mouse_Q8BX 02 _ $93.5 \%$ Rattus norvegicus rat D4AG04_91.18 Bos taurus_bovin_F1N4W0_92.98 Equus ferus cabaillus_horse_F7DLU4_848 Rhinolophusferrumequinum bat AOA 94.871 FWH 77.2 Terrapene carolina triunguis turt le A0A $674 \mathrm{~J} 491$ _ 80.03 Xenopus tropicalis frog A8WGV7 70.8 Danio rerio_zebrafish_E90H 65 _61.5 Drosophila melanogaster fruitfly_A0A034WFO0 35.6\% Caenorhabditis elegans_round worm_019518_24.48 Saccharomyces cerevisiae_budding yeast_P38875_29.0

448 RALLKNTEYT PDENHGFYV 448 RALLKNTEYT FDENHGFYV

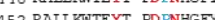
468 RALI XNTEYT DDNAGG 448 RALIWTTEYT DDENHGFY 521 RALTZNTEYT DDPNHGFY 448 RALLKNTEYT FDENHGFY 346 RALLKNTEYT PDENHGF 447 RALLKNTEYT FDENHGFY 448 RALLKNTEYP PDENHGFY 504 RALLKNTEYT EDENHGF 444 YIFLKNLEYY EDANHGHYL 401 KQLLRLEQYP FDANHGMII 495 KALLOFAEYP PDANHGFII 438 KTPLRLDEYP FDANRGYN

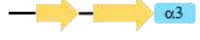

Fig. S7. The GPI-binding site is delineated with conserved residues and hosts a

digitonin molecule. a A digitonin (light blue) which also contains a polyglycan chain is found near GPI (green). The cryo-EM density of the digitonin is shown in the right

515 VNLPTPDFSM PYNVICLTC 515 VNLPTPDFSM PYNVICLTC 519 VNLPTPDFSM PYNVICLTC 535 VNLPTPDESM PYNVICLTC 588 VNLPTPDESM PYNVICLTC 515 VSLPTPDFSM PYNVICLTC 413 VSLPTPDFSM PYNVICLTC 515 VNLPTPDESM PYNVICLTC 571 VNLPTPDESM PYNVICLTC 509 LSLPTPDFSM PYNVICLAC 539 LSLSTPDFSM PYNVIILTS 481 MFIPTPDFSM PYNVIIFTS TMHT1 corner. b Apparent activity of GPI-binding site mutants relative to the wildtype (WT). Activity was measured by immune staining of a reporter GPI-AP(CD59) on the surface of KO cells transfected with the indicated mutants. Data represent mean \pm s.e.m. from three independent experiments (Supplementary Data 1). Activity for PIGT D521/S523, GPAA1 H354, and PIGU N385 are re-plotted using data from Fig. 6d. c, d Sequence alignment of the conserved residues of PIGK (c) and PIGT (d) around the GPI-binding site (Fig. 6b). Protein sequences were selected from evolutionarily 
representative species. Uniprot IDs of the PIGT/PIGK sequences and their sequence identity to the human ortholog were shown along with the binomial nomenclature and common names. Asterisk, colon, and dot indicate identical, conserved, and semiconserved substitutions, respectively. Secondary elements were labeled for easier location of the corresponding elements in Fig. S2. 

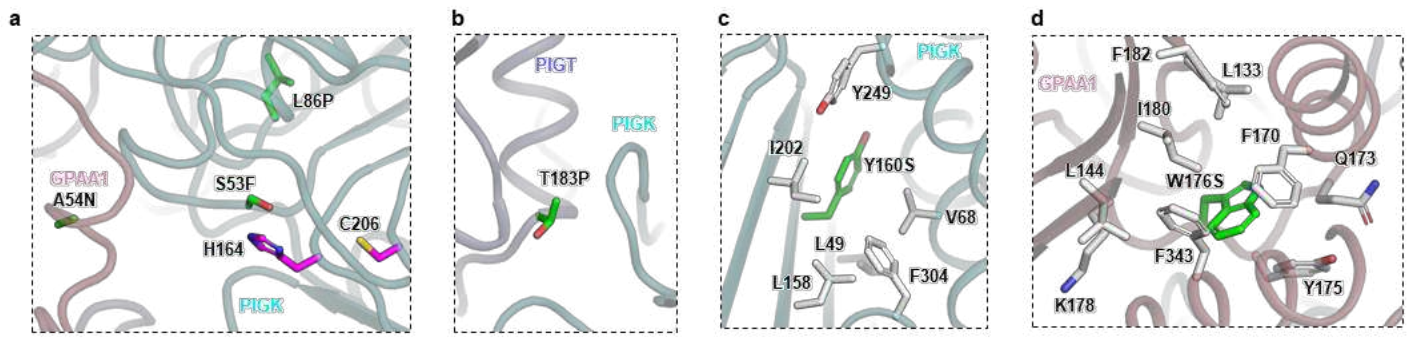

Fig. S8. Expanded view of the distribution of representative genetic mutations. a,

b Exemplary expanded view of residues close to the catalytic dyad (a) and at the interpocket. 
a

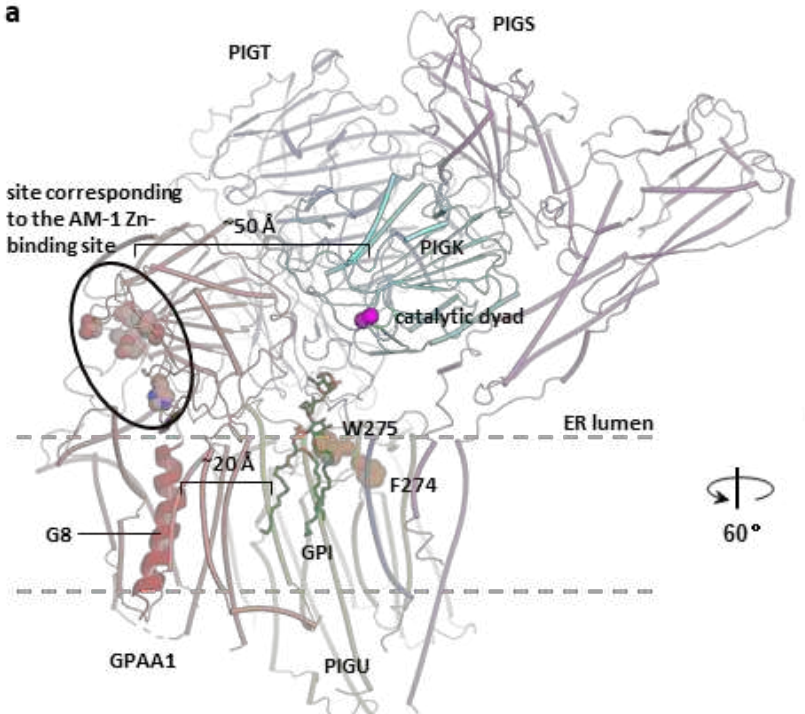

c d Expanded view. b

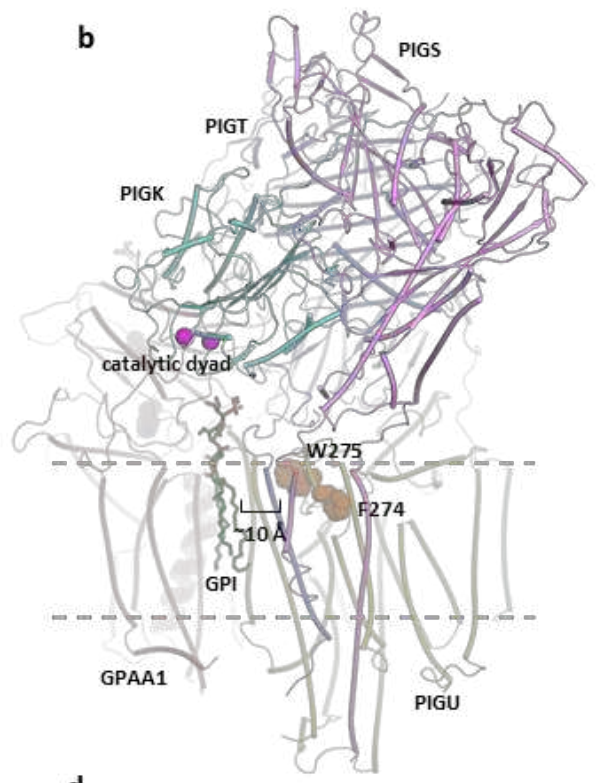

d

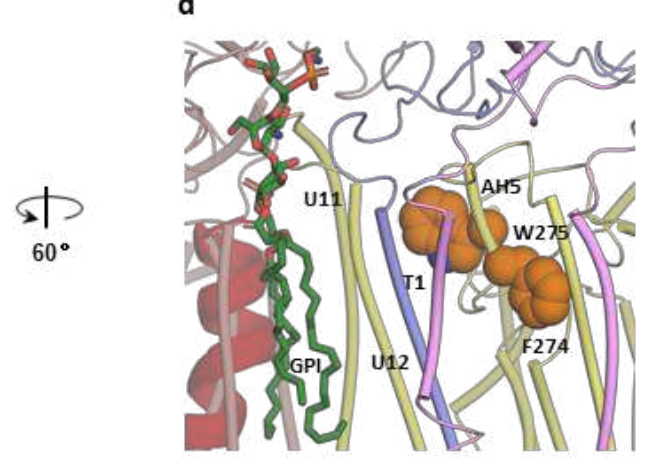

Fig. S9. The site corresponding to the AM-1 Zn-binding site, the TMH8 of GPAA1, and F274/W275 in PIGU are not at the proposed active site. Various components are labeled appropriately. The catalytic dyad is shown as $\mathrm{C} \alpha$ spheres. a, b Overview. c, 


\section{Human GPI-T complex}

\section{Data collection and processing}

Magnification

Voltage $(\mathrm{kV})$

Electron exposure $\left(\mathrm{e}^{-} / \AA^{2}\right)$

Defocus range $(\mu \mathrm{m})$

Pixel size $(\AA)$

Symmetry imposed

Initial particle images (no.)

Final particle images (no.)

Map resolution $(\AA)$

FSC threshold

Map resolution range $(\AA)$
165,000

300

52.5

-2.4 to -0.6

0.85

C1

$2,959,791$

151,509

2.53

0.143

$2.3-3.9$

Model resolution $(\AA)$

FSC threshold

0.5

Map sharpening $B$ factor $\left(\AA^{2}\right)$ 77.5

Model composition

Non-hydrogen atoms

19,733 
Protein residues

2,393

Ligands

$B$ factor $\left(\AA^{2}\right)$

Protein

50.8

Ligand

64.8

R.m.s. deviations

Bond lengths ( $\mathrm{A})$

0.019

Bond angles $\left({ }^{\circ}\right)$

1.325

Ramachandran plot

Favored (\%)

97.77

Allowed (\%)

2.15

Outliers (\%)

0.08 


\section{References}

1. Gamage, D.G. \& Hendrickson, T.L. GPI transamidase and GPI anchored proteins: oncogenes and biomarkers for cancer. Crit Rev Biochem Mol Biol 48, 446-64 (2013).

2. Pittet, M. \& Conzelmann, A. Biosynthesis and function of GPI proteins in the yeast Saccharomyces cerevisiae. Biochim Biophys Acta 1771, 405-20 (2007).

3. Kinoshita, T. Biosynthesis and biology of mammalian GPI-anchored proteins. Open Biol 10, 190290 (2020).

4. Peter, O. \& Menon, A.K. Thematic review series: Lipid Posttranslational Modifications. GPI anchoring of protein in yeast and mammalian cells, or: how we learned to stop worrying and love glycophospholipids. J Lipid Res 48, 993-1011 (2007).

5. Paulick, M.G. \& Bertozzi, C.R. The Glycosylphosphatidylinositol Anchor: A Complex Membrane-Anchoring Structure for Proteins. Biochemistry 47, 6991-7000 (2008).

6. Ferguson, M.A.J., Homans, S.W., Dwek, R.A. \& Rademacher, T.W. Glycosyl-Phosphatidylinositol Moiety That Anchors Trypanosoma brucei Variant Surface Glycoprotein to the Membrane. Science 239, 753-759 (1988).

7. Schofield, L., Hewitt, M.C., Evans, K., Siomos, M.A. \& Seeberger, P.H. Synthetic GPI as a candidate anti-toxic vaccine in a model of malaria. Nature 418, 785-9 (2002).

8. Fujihara, Y. et al. Expression of TEX101, regulated by ACE, is essential for the production of fertile mouse spermatozoa. Proc Natl Acad Sci USA 110, 8111-8116 (2013).

9. Filmus, J. \& Capurro, M. The role of glypicans in Hedgehog signaling. Matrix Biol 35, 248-252 (2014).

10. Cho, C., Wang, Y., Smallwood, P.M., Williams, J. \& Nathans, J. Molecular determinants in Frizzled, Reck, and Wnt7a for ligand-specific signaling in neurovascular development. eLife 8, e47300 (2019).

11. Lubbers, R., van Essen, M.F., van Kooten, C. \& Trouw, L.A. Production of complement components by cells of the immune system. Clin Exp Immunol 188, 183-194 (2017).

12. Nsabimana, A. et al. Alkaline phosphatase-based electrochemical sensors for health applications. Anal Methods 11, 1996-2006 (2019).

13. Scaranti, M., Cojocaru, E., Banerjee, S. \& Banerji, U. Exploiting the folate receptor $\alpha$ in oncology. Nat Rev Clin Oncol 17, 349-359 (2020).

14. McKean, D.M. \& Niswander, L. Defects in GPI biosynthesis perturb Cripto signaling during forebrain development in two new mouse models of holoprosencephaly. Biology Open $\mathbf{1}$ 874-883 (2012).

15. Smith, T.K., Crossman, A., Brimacombe, J.S. \& Ferguson, M.A.J. Chemical validation of GPI biosynthesis as a drug target against African sleeping sickness. EMBO J 23, 4701-4708 (2004).

16. Hong, Y. et al. Human PIG-U and Yeast Cdc91p Are the Fifth Subunit of GPI Transamidase That Attaches GPI-Anchors to Proteins. Mol Biol Cell 14, 1780-1789 (2003).

17. Meyer, U., Benghezal, M., Imhof, I. \& Conzelmann, A. Active Site Determination of Gpi8p, a Caspase-Related Enzyme Required for Glycosylphosphatidylinositol Anchor Addition to Proteins. Biochemistry 39, 3461-3471 (2000).

18. Ohishi, K. et al. Gaa1p and Gpi8p Are Components of a Glycosylphosphatidylinositol (GPI) Transamidase That Mediates Attachment of GPI to Proteins. Mol Biol Cell 11, 1523-1533 (2000). 
19. Eisenhaber, B., Eisenhaber, S., Kwang, T.Y., Grüber, G. \& Eisenhaber, F. Transamidase subunit GAA1/GPAA1 is a M28 family metallo-peptide-synthetase that catalyzes the peptide bond formation between the substrate protein's omega-site and the GPI lipid anchor's phosphoethanolamine. Cell Cycle 13, 1912-1917 (2014).

20. Vainauskas, S., Maeda, Y., Kurniawan, H., Kinoshita, T. \& Menon, A.K. Structural Requirements for the Recruitment of Gaa1 into a Functional Glycosylphosphatidylinositol Transamidase Complex. J Biol Chem 277, 30535-30542 (2002).

21. Ohishi, K., Nagamune, K., Maeda, Y. \& Kinoshita, T. Two subunits of glycosylphosphatidylinositol transamidase, GPI8 and PIG-T, form a functionally important intermolecular disulfide bridge. J Biol Chem 278, 13959-67 (2003).

22. Ohishi, K., Inoue, N. \& Kinoshita, T. PIG-S and PIG-T, essential for GPI anchor attachment to proteins, form a complex with GAA1 and GPI8. EMBO J 20, 4088-98 (2001).

23. Sharma, D.K. et al. Soluble GPI8 restores glycosylphosphatidylinositol anchoring in a trypanosome cell-free system depleted of lumenal endoplasmic reticulum proteins. Biochem J 351 Pt 3, 717-22 (2000).

24. Nguyen, T.T.M. et al. Bi-allelic Variants in the GPI Transamidase Subunit PIGK Cause a Neurodevelopmental Syndrome with Hypotonia, Cerebellar Atrophy, and Epilepsy. Am J Hum Genet 106, 484-495 (2020).

25. Knaus, A. et al. Mutations in PIGU Impair the Function of the GPI Transamidase Complex, Causing Severe Intellectual Disability, Epilepsy, and Brain Anomalies. Am J Hum Genet 105, 395-402 (2019).

26. Nguyen, T.T.M. et al. Mutations in PIGS, Encoding a GPI Transamidase, Cause a Neurological Syndrome Ranging from Fetal Akinesia to Epileptic Encephalopathy. Am J Hum Genet 103, 602-611 (2018)

27. Kvarnung, M. et al. A novel intellectual disability syndrome caused by GPI anchor deficiency due to homozygous mutations in PIGT. J Med Genet 50, 521-528 (2013).

28. Yang, L. et al. Homozygous PIGT Mutation Lead to Multiple Congenital Anomalies-Hypotonia Seizures Syndrome 3. Frontiers in Genetics 9(2018).

29. Pagnamenta, A.T. et al. Analysis of exome data for 4293 trios suggests GPI-anchor biogenesis defects are a rare cause of developmental disorders. Eur J Hum Genet 25, 669-679 (2017).

30. Kohashi, K. et al. Epileptic apnea in a patient with inherited glycosylphosphatidylinositol anchor deficiency and PIGT mutations. Brain Dev 40, 53-57 (2018).

31. Skauli, N. et al. Novel PIGT Variant in Two Brothers: Expansion of the Multiple Congenital Anomalies-Hypotonia Seizures Syndrome 3 Phenotype. Genes (Basel) 7(2016).

32. Nakashima, M. et al. Novel compound heterozygous PIGT mutations caused multiple congenital anomalies-hypotonia-seizures syndrome 3. Neurogenetics 15, 193-200 (2014).

33. Nguyen, T.T.M. et al. Mutations in GPAA1, Encoding a GPI Transamidase Complex Protein, Cause Developmental Delay, Epilepsy, Cerebellar Atrophy, and Osteopenia. Am J Hum Genet 101, 856-865 (2017).

34. Yi, L. et al. Disulfide Bond Formation and N-Glycosylation Modulate Protein-Protein Interactions in GPI-Transamidase (GPIT). Sci Rep 8, 45912 (2017).

35. Hong, Y. et al. TbGPI16 is an essential component of GPI transamidase in Trypanosoma brucei. FEBS Letters 580, 603-606 (2006).

36. Eisenhaber, B., Sinha, S., Wong, W.C. \& Eisenhaber, F. Function of a membrane-embedded 
domain evolutionarily multiplied in the GPI lipid anchor pathway proteins PIG-B, PIG-M, PIGU, PIG-W, PIG-V, and PIG-Z. Cell Cycle 17, 874-880 (2018).

37. Cai, H. et al. An improved fluorescent tag and its nanobodies for membrane protein expression, stability assay, and purification. Commun Biol 3, 753 (2020).

38. Fuentes-Prior, P. \& Salvesen, G.S. The protein structures that shape caspase activity, specificity, activation and inhibition. Biochem J 384, 201-32 (2004).

39. Nonis, S.G. et al. Structural and biochemical analyses of concanavalin A circular permutation by jack bean asparaginyl endopeptidase. Plant Cell 33, 2794-2811 (2021).

40. Dall, E. \& Brandstetter, H. Mechanistic and structural studies on legumain explain its zymogenicity, distinct activation pathways, and regulation. Proc Natl Acad Sci USA 110, 10940-10945 (2013).

41. Holm, L. \& Rosenström, P. Dali server: conservation mapping in 3D. Nucleic Acids Res 38, W545-W549 (2010).

42. Waltersperger, S., Widmer, C., Wang, M. \& Baumann, U. Crystal structure of archaemetzincin amza from Methanopyrus kandleri at $1.5 \AA$ Å resolution. Proteins 78, 2720-2723 (2010).

43. Lu, P. et al. Three-dimensional structure of human $\gamma$-secretase. Nature 512, 166-170 (2014).

44. Zimmer, J. Structural features underlying recognition and translocation of extracellular polysaccharides. Interface Focus 9, 20180060 (2019).

45. Fraering, P. et al. The GPI transamidase complex of Saccharomyces cerevisiae contains Gaa1p, Gpi8p, and Gpi16p. Mol Biol Cell 12, 3295-3306 (2001).

46. Burr, M.L. et al. CMTM6 maintains the expression of PD-L1 and regulates anti-tumour immunity. Nature 549, 101-105 (2017).

47. Qian, H. et al. Inhibition of tetrameric Patched1 by Sonic Hedgehog through an asymmetric paradigm. Nature Communications 10, 2320 (2019).

48. Reading, E. et al. The Effect of Detergent, Temperature, and Lipid on the Oligomeric State of MscL Constructs: Insights from Mass Spectrometry. Chemistry \& biology 22, 593-603 (2015).

49. Neumann, B., Chang, C.C.Y. \& Chang, T.-Y. A simple method to disrupt and restore subunit interaction of acyl-CoA:cholesterol acyltransferase 1. MethodsX 6, 2242-2247 (2019).

50. Dall, E., Fegg, J.C., Briza, P. \& Brandstetter, H. Structure and mechanism of an aspartimidedependent peptide ligase in human legumain. Angew Chem Int Ed 54, 2917-2921 (2015).

51. Jacobitz, A.W., Kattke, M.D., Wereszczynski, J. \& Clubb, R.T. Sortase Transpeptidases: Structural Biology and Catalytic Mechanism. Adv Protein Chem Struct Biol 109, 223-264 (2017).

52. Gibson, D.G. et al. Complete Chemical Synthesis, Assembly, and Cloning of a <i>Mycoplasma genitalium</i> Genome. Science 319, 1215-1220 (2008).

53. $\mathrm{Xu}, \mathrm{H}$. et al. Sequence determinants of improved CRISPR sgRNA design. Genome Res 25, 1147-57 (2015).

54. Zivanov, J. et al. New tools for automated high-resolution cryo-EM structure determination in RELION-3. elife 7, e42166 (2018).

55. Punjani, A., Rubinstein, J.L., Fleet, D.J. \& Brubaker, M.A. cryoSPARC: algorithms for rapid unsupervised cryo-EM structure determination. Nature Methods 14, 290-296 (2017).

56. Zheng, S.Q. et al. MotionCor2: anisotropic correction of beam-induced motion for improved cryo-electron microscopy. Nature Methods 14, 331-332 (2017).

57. Rohou, A. \& Grigorieff, N. CTFFIND4: Fast and accurate defocus estimation from electron 
micrographs. Journal of Structural Biology 192, 216-221 (2015).

58. Emsley, P., Lohkamp, B., Scott, W.G. \& Cowtan, K. Features and development of Coot. Acta Crystallogr D Biol Crystallogr 66, 486-501 (2010).

59. Afonine, P.V. et al. Real-space refinement in PHENIX for cryo-EM and crystallography. Acta Crystallogr D Struct Biol 74, 531-544 (2018).

60. Pettersen, E.F. et al. UCSF ChimeraX: Structure visualization for researchers, educators, and developers. Protein Sci 30, 70-82 (2021). 


\section{Supplementary Files}

This is a list of supplementary files associated with this preprint. Click to download.

- FACSdataGPITmutations20220219.xIsx 